\title{
SEMI-GROUPS OF MEASURES ON LIE GROUPS
}

\author{
BY \\ G. A. HUNT( ${ }^{(1)}$
}

This paper grew out of discussions with S. Bochner. It would be hard now to disengage his contributions from mine.

We shall characterize those families $\left(p_{t}\right)_{0<t<\infty}$ of finite positive measures on a Lie group $G$ which are weakly continuous and form a semi-group under convolution. No generality is lost in assuming that the $p_{t}$ are probability measures.

Paul Lévy [3] obtained a characterization when $G$ is $R$, the additive group of reals: The characteristic function $\phi_{t}(\xi)=\int e^{i \xi \sigma} p_{t}(d \sigma)$ has the form

$$
\phi_{t}(\xi)=\exp \left\{i t a \xi-t b \xi^{2}+t \int\left(e^{i \xi \sigma}-1-\frac{i \xi \sigma}{1+\sigma^{2}}\right) G(d \sigma)\right\}
$$

where $b$ is non-negative and $G$ is a positive measure on $R$ such that $G(0)=0$ and $\int \sigma^{2} G(d \sigma)$ is finite.

Let us translate this result. The definition $S_{t} f \cdot(\tau)=\int f(\tau+\sigma) p_{t}(d \sigma)$ yields a semi-group $\left(S_{t}\right)$ of transformations which are defined at least for bounded continuous functions. It is easy to prove that the limit

$$
\begin{aligned}
M f \cdot(\tau) & =\lim _{t \supset 0} \frac{1}{t}\left[S_{t} f \cdot(\tau)-f(\tau)\right] \\
& =a f^{\prime}(\tau)+b f^{\prime \prime}(\tau)+\int\left(f(\tau+\sigma)-f(\tau)-\frac{f^{\prime}(\tau) \sigma}{1+\sigma^{2}}\right) G(d \sigma)
\end{aligned}
$$

exists if $f$ is the Fourier transform of a function vanishing rapidly at infinity. Moreover, $M$ determines the family $\left(p_{t}\right)$.

This second form extends to a semi-group $\left(p_{t}\right)$ on a Lie group $G$. Let us first assume

$$
\lim _{\triangle \backslash 0} p_{t}(E)=1, \quad \text { E a neighborhood of } \epsilon,
$$

(here $\epsilon$ is the neutral element). Then the $p_{t}$ define a semi-group $\left(S_{t}\right)$ of transformations on a Banach space $\mathcal{F}$ of functions with the property that $S_{f} f \rightarrow f$ as $t$ decreases to 0 . Thus $\left(S_{t}\right)$ has an infinitesimal generator; one would expect it to have an expression like (2).

Received by the editors June 4, 1955 .

(1) This research was supported by the United States Air Force through the Office of Scientific Research of the Air Research and Development Command. 
The first part of this paper proves that this is indeed so when $\mathcal{F}$ is the space of continuous functions on the one point compactification of $G$. The subject proper is begun in $\$ 2$ after disposing of a good many preliminaries in $\S 1$. The main argument is carried out in $\S \S 3,4$ and the results are summarized in $\$ 5$, especially in Theorem 5.1. This choice of $\mathcal{F}$ facilitates the proofs; but it has the drawback that $\mathcal{F}$, though adequate for the characterization of $\left(p_{t}\right)$, is rather small-for example, when $G$ is $R$ it does not contain the characters $e^{i \xi \sigma}$, so that Lévy's formula is not an immediate consequence of Theorem 5.1.

The second part brings two complements. First, in $\$ 6$ we prove a version of Theorem 5.1 with another choice of $\mathcal{F}$; the new statement implies Lévy's formula at once. Next, we deal briefly with the $L^{2}$ theory in $\$ 7$, showing for example that the $p_{t}$ define self-adjoint operators if and only if $M$ has a certain symmetry.

The last part deals with the $\left(p_{t}\right)$ which do not satisfy (3). It turns out (\$10) that as $t$ decreases the $p_{t}$ tend weakly to the Haar measure of a compact subgroup $K$ of $G$ and that the homogeneous space $G / K$ is the proper place to look for a semi-group representing $\left(p_{t}\right)$. We begin by laying the necessary foundation in $\S 8$. Then $\S 9$ states the analogue of Theorem 5.1 and sketches the proof. The results are translated back to $G$ in $\S 10$. What we have said in $\S \S 6,7$ can also be carried over to homogeneous spaces.

One could present matters in the reverse order. I have not done so for two reasons. The preliminaries, which are long already, would then delay too much the coming to grips with the problem. Next, the expression for the infinitesimal generator under the hypothesis (3) is a little more satisfactory than the one in $\S 9$.

Lévy's result is more complete than I have indicated. He showed that an infinitely divisible measure on $R$ may always be taken to be $p_{1}$ in a semigroup $\left(p_{t}\right)$. This statement and its extension to Abelian $G$ can be fairly easily derived from Theorem 5.1. Simple examples show that when $G$ is not Abelian some restriction must be placed upon the infinitely divisible measure $\mu$. For $G$ compact it suffices to assume that $\mu$ factors into parts which commute one with another. I have not included the proof because it rests on the fact that $G$ has sufficiently many finite-dimensional representations and does not carry over to arbitrary Lie groups.

\section{Semi-groups on $G$}

1. Preliminaries. This section deals with matters which otherwise would interrupt the argument. $\$ \$ 1.1-1.5$ fix the notation and remind the reader of those facts which will be used without mention. $\$ 1.6$ shows how to approximate certain differential operators. $\$ 1.7$ summarizes what is needed from the theory of semi-groups of transformations. And $\$ 1.8$ proves, in the generality we require, a lemma familiar from the theory of parabolic differential equations. 
1.1. $G$ is a Lie group with neutral element $\epsilon$. If $G$ is not compact $G_{c}$ is its one point compactification. We shall always suppose that a homeomorphism of $G$ onto $G$ is extended to $G_{c}$ by making the point $\omega$ at infinity correspond to itself; accordingly we set $\sigma \omega=\omega \sigma=\omega$ for every $\sigma$ in $G_{c}$. If $G$ is compact take $G_{c}$ to be $G$ itself; in this case all statements concerning $\omega$ are to be disregarded.

$\mathcal{C}$ is the Banach space of continuous functions on $G_{c}$, with norm $\|f\|$ the maximum of $|f(\sigma)|$. A function belonging to $\mathcal{C}$ is of course uniformly continuous in both the right and left uniform structures on $G$. For $\sigma$ in $G$ the translations $R_{\sigma}$ and $L_{\sigma}$ of $\mathcal{C}$ are defined by

$$
R_{\sigma} f \cdot(\tau)=f(\tau \sigma), \quad L_{\sigma} f \cdot(\tau)=f\left(\sigma^{-1} \tau\right), \quad \tau \in G_{c},
$$

where $R_{\sigma} f \cdot(\tau)$ denotes the value of the function $R_{\sigma} f$ at $\tau$.

1.2. The space of finite measures on the Borel sets of $G_{c}$ is given the usual weak topology. Thus the sets comprising those measures $\mu$ for which $a<\int f(\sigma) \mu(d \sigma)<b$ form a sub-basis for the topology; here $f$ ranges over $\mathcal{C}$ and $a, b$ over the reals.

If $\mu$ is a finite measure the associated transformation $R_{\mu}$,

$$
R_{\mu} f \cdot(\tau)=\int_{G_{c}} f(\tau \sigma) \mu(d \sigma), \quad f \in \mathcal{C},
$$

is a continuous linear transformation of $\mathcal{C}$ into itself whose bound is the total variation of $\mu$. The convolution $\mu * \nu$ is defined by

$$
\begin{array}{ll}
\mu * \nu \cdot(E)=\int_{G} \mu(d \sigma) \nu\left(\sigma^{-1} E\right), & E \subset G, \\
\mu * \nu \cdot(\omega) & =\mu(\omega) \nu\left(G_{c}\right)+\nu(\omega) \mu(G) .
\end{array}
$$

The last equation reduces to $\mu * \nu \cdot(\omega)=\mu(\omega)+\nu(\omega)-\mu(\omega) \nu(\omega)$ if both $\mu$ and $\nu$ are probability measures. Convolution is associative and $R_{\mu * \nu}=R_{\mu} R_{\nu}$.

We shall be concerned only with positive measures. It can be shown that the variable positive measure $\mu$ tends to the measure $\nu$ if and only if the associated transformation $R_{\mu}$ tends strongly to $R_{\nu}$, provided that $\mu(\omega)$ tends to $\nu(\omega)$. It follows that $\mu * \nu$ is continuous in the pair $(\mu, \nu)$ if both $\mu$ and $\nu$ are positive and if $\mu(\omega)$ and $\nu(\omega)$ vary continuously.

1.3. Let $Y$ be an element of the left invariant Lie algebra of $G$ and set $\zeta(s)=\exp s Y$. If $f$ is in $C$ define

$$
Y f=\lim _{s \rightarrow 0} \frac{1}{s}\left(R_{\zeta(s)} f-f\right)
$$

provided the limit exists in the metric of $\mathcal{C}$. Note that $Y f \cdot(\omega)$ must vanish. $\mathcal{C}_{k}$ is the set of those $f$ for which $Y_{1}\left(Y_{2} \cdots\left(Y_{k} f\right) \cdots\right)$ makes sense for every choice of the $Y_{i}$ in the Lie algebra. $\mathcal{C}_{k}$ is a dense linear manifold in $\mathcal{C}$, for it 
includes every function which is sufficiently differentiable on $G$ and constant near $\omega$. Let us take once for all a basis $X_{1}, \cdots, X_{d}$ of the Lie algebra and define on $\mathcal{C}_{k}$ the norm

$$
\|f\|_{k}=\|f\|+\sum_{i}\left\|X_{i} f\right\|+\cdots+\sum_{i_{1}, \cdots, i_{k}}\left\|X_{i_{1}} \cdots X_{i_{k}} f\right\|,
$$

thus making $\mathcal{C}_{k}$ a Banach space. Left translations leave $\mathcal{C}_{k}$ invariant, and $\left\|L_{\sigma} f-f\right\|_{k} \rightarrow 0$ as $\sigma \rightarrow \epsilon$, provided $f$ belongs to $\mathcal{C}_{k}$.

1.4. In several proofs we shall use the space $\mathcal{C}_{k}^{\prime}$, whose definition parallels that of $\mathcal{C}_{k}$. With $Y$ in the Lie algebra of $G$ and $\zeta(s)=\exp s Y$ define

$$
Y^{\prime} f=\lim _{s \rightarrow 0} \frac{1}{s}\left(L_{\zeta(s)} f-f\right)
$$

whenever $f$ is in $\mathcal{C}$ and the limit exists in the metric of $\mathcal{C}$. Then $\mathcal{C}_{k}^{\prime}$ and $\|f\|_{k}^{\prime}$ are defined just as $\mathcal{C}_{k}$ and $\|f\|_{k}$ were before. $\mathcal{C}_{k}^{\prime}$ is invariant under right translations. Of course, $\mathcal{C}_{k}$ and $\mathcal{C}_{k}^{\prime}$ coincide if $G$ is compact or Abelian.

1.5. We shall be particularly concerned with differential operators of the form

$$
\begin{aligned}
\Delta f & =\sum a_{i} X_{i} f+\sum a_{i j} X_{i} X_{j} f \\
& =\Delta_{1} f+\Delta_{2} f,
\end{aligned}
$$$$
f \in \mathcal{C}_{2},
$$

where the $a_{i}$ and $a_{i j}$ are constants. We assume that $a_{i j}=a_{j i}$, for terms $\left(a_{i j}-a_{j i}\right)\left(X_{i} X_{j}-X_{j} X_{i}\right)$ are elements of the Lie algebra and may be adsorbed into the first sum; with this restriction the representation of $\Delta$ is unique once the basis $X_{i}$ is given.

Let $\rho$ be the right invariant Haar measure on $G$. If $f$ and $g$ belong to $\rho_{1}$ and vanish near $\omega$ then

$$
\int_{G} Y f \cdot(\sigma) g(\sigma) \rho(d \sigma)=-\int_{G} f(\sigma) Y g \cdot(\sigma) \rho(d \sigma)
$$

for every $Y$ in the Lie algebra of $G$. It follows that

$$
\begin{aligned}
& \int \Delta_{1} f \cdot(\sigma) g(\sigma) \rho(d \sigma)=-\int f(\sigma) \Delta_{1} g \cdot(\sigma) \rho(d \sigma), \\
& \int \Delta_{2} f \cdot(\sigma) g(\sigma) \rho(d \sigma)=\int f(\sigma) \Delta_{2} g \cdot(\sigma) \rho(d \sigma)
\end{aligned}
$$

if $f$ and $g$ are in $\mathcal{C}_{2}$ and vanish near $\omega$. Thus $\Delta_{2}$ is formally self-adjoint with respect to $\rho$ and the decomposition $\Delta=\Delta_{1}+\Delta_{2}$ is quite independent of the basis $X_{i}$.

1.6. Consider a $\Delta$ for which the matrix $\left(a_{i j}\right)$ is positive semi-definite. In another basis we may write 


$$
\Delta=\Delta_{1}+\sum_{1 \leqq i \leqq l} Y_{i} Y_{i}
$$

where $l$ is some integer between 0 and $d$. Now let $\zeta_{s}=\exp s \Delta_{1}$ and $\zeta_{s}^{i}=\exp s Y_{i}$. Then for every $f$ in $C_{2}$

$$
\begin{aligned}
\Delta f \cdot(\tau)= & \lim _{s \rightarrow 0} \frac{1}{s}\left[f\left(\tau \zeta_{s}\right)-f(\tau)\right] \\
& +\lim _{s \rightarrow 0} \frac{1}{s^{2}} \sum_{1 \leqq i \leqq l}\left[f\left(\tau \zeta_{s}^{i}\right)-2 f(\tau)+f\left(\tau \zeta_{-s}^{i}\right)\right]
\end{aligned}
$$

and the limits exist uniformly for $\tau$ in $G_{c}$. On taking into account the form of the coefficients on the right, we see that there is a sequence of finite positive measures $\mu_{n}$ on $G-\{\epsilon\}$ such that

$$
\Delta f \cdot(\tau)=\lim _{n \rightarrow \infty} \int_{\mathcal{G}^{-\{\epsilon\}}}[f(\tau \sigma)-f(\tau)] \mu_{n}(d \sigma),
$$

the limit being in the metric of $\mathcal{C}$ provided $f$ is in $\mathcal{C}_{2}$. This result will be used in $\S 4$.

1.7. Let $\Upsilon$ be a Banach space and $\left(T_{t}\right)_{0<t<\infty}$ a semi-group of linear transformations on $\Upsilon$. Under the hypotheses

$$
\begin{array}{rlr}
\left\|T_{t}\right\| & \leqq 1, & 0<t<\infty, \\
\lim _{\lambda 0} T_{\imath} y & =y, & y \in r,
\end{array}
$$

the limit

$$
N y=\lim _{t>0} \frac{1}{t}\left(T_{t} y-y\right)
$$

exists on a dense linear subset $\mathcal{D}$ of $\Upsilon$. The operator $N$, defined on $\mathcal{D}$ by (2), is the infinitesimal generator of the semi-group $\left(T_{t}\right)$, and

$$
T_{\imath} y-y=\int_{0}^{t} T_{8} N y d s
$$

for $y$ in $\mathcal{D}$.

1.8. Let $X$ be a compact space, $I$ the interval $0 \leqq t<\infty$, and $f$ a continuous function on $I \times X$. Moreover, let $f$ have the property that at those points $(s, y)$ of $I \times X$ satisfying

$$
s>0, \quad f(s, y)=\min _{x \in X} f(s, x)
$$

the derivative $\partial f(s, y) / \partial t$ exists and is non-negative. Then $\min _{x} f(t, x)$ is at least $\min _{x} f(0, x)$ for all $t$. 
In the proof we may add a constant to $f$ and take $\min f(0, x)=1$. Let $c$ be positive and consider the function $g(t, x)=e^{c t} f(t, x)$, which achieves its minimum for a given $t$ at the same points of $X$ as does $f(t, x)$. If $g$ is somewhere less than 1 there exist $\delta, s, y$ such that $0<\delta<1, s>0, g(s, y)=\delta$, and $g(t, x)>\delta$ for all $x$ and all $t$ less than $s$. Consequently $\partial g(s, y) / \partial t$ is not positive. On the other hand $g(s, y)=\min _{x} g(s, x)$, so that $f(s, y)=\min _{x} f(s, x)$ and

$$
\begin{aligned}
\frac{\partial g}{\partial t}(s, y) & =e^{c s} \frac{\partial f}{\partial t}(s, y)+c g(s, y) \\
& \geqq c \delta>0,
\end{aligned}
$$

and we have a contradiction. Therefore $g$ is never less than 1 ; letting $c$ decrease to 0 we obtain the same result for $f$.

Now let the Banach space $\Upsilon$ of $\S 1.7$ be the space $\mathcal{C}(X)$ of continuous functions on $X$ and $\left(T_{t}\right)$ a semi-group which satisfies (1). We keep the notation of $\$ 1.7$.

Lemma. The semi-group $\left(T_{t}\right)$ has the property

$$
\min _{x \in X} T_{t} f \cdot(x) \geqq \min _{x \in X} f(x), \quad f \in \mathcal{C}(\mathcal{X}), t>0,
$$

if and only if

$$
N f \cdot(y) \geqq 0 \text { whenever } f \in \mathcal{D} \text { and } f(y)=\min _{x \in X} f(x) .
$$

That (3) implies (4) follows from the definition of $N$. Note that $d T_{t} f / d t$ coincides with $N T_{t} f$ for all $f$ and all positive $t$ whenever $f$ belongs to $\mathscr{D}$. Thus if (4) is true and $f \in \mathcal{D}$ the function $f(t, x)=T_{t} f \cdot(x)$ fulfills the conditions at the beginning of this paragraph, so that (3) must hold. Then (3) is true for all $f$ because $\mathcal{D}$ is dense in $\mathcal{C}(X)$.

2. Positive semi-groups. Let $\left(p_{t}\right)_{0<t<\infty}$ be a family of positive measures on the Borel sets of $G_{c}$ which form a semi-group under convolution and satisfy

$$
\begin{aligned}
p_{t}\left(G_{c}\right) & =1, \\
\lim _{t>0} p_{t}(E) & =1,
\end{aligned} \quad \begin{aligned}
t & >0,
\end{aligned}
$$

It follows from (2) that the family is continuous in the weak topology of measures. Also the definition of convolution implies that $p_{t}(G)$ is a bounded solution of the functional equation $u(s+t)=u(s) u(t)$, so that $p_{t}(\omega)=1-p_{t}(G)$ varies continuously.

2.1. The measures $p_{t}$ give rise to a strongly continuous semi-group of transformations $S_{t}$ of the Banach space $\mathcal{C}$ :

$$
S_{t} f \cdot(\tau)=\int_{G_{c}} f(\tau \sigma) p_{t}(d \sigma), \quad f \in \mathcal{C}, \tau \in G_{c} .
$$


This definition and the properties of the $p_{t}$ imply that

$$
\begin{aligned}
\lim _{i>0} S_{t} f & =f, & f \in \mathcal{C}, \\
\min _{\sigma \in G_{c}} S_{t} f \cdot(\sigma) & \geqq \min _{\sigma \in \mathcal{G}_{c}} f(\sigma), & t>0, f \in \mathcal{C}, \\
L_{\sigma} S_{t} & =S_{t} L_{\sigma}, & t>0, \sigma \in \mathcal{G} .
\end{aligned}
$$

The inequality (5) is equivalent to the statement that $S_{t}$ leaves constants invariant and transforms positive functions into positive functions.

2.2. Conversely, suppose we are given a semi-group of linear transformations $S_{t}$ of $\mathcal{C}$ for which (4), (5), and (6) are true. From (5) it follows that the bound of each $S_{t}$ is 1 and that for each positive $t$ and each $\tau$ in $G_{c}$,

$$
S_{t} f \cdot(\tau)=\int_{G_{c}} f(\sigma) q_{t}(\tau, d \sigma)
$$

with $q_{t}(\tau, E)$ a positive measure of total measure 1. Then (6) implies that $q_{t}(\sigma \tau, \sigma E)=q_{t}(\tau, E)$ for $\sigma$ in $G$ and $\tau$ in $G_{c}$; so there is a measure $p_{t}(E)$ $=q_{t}(\epsilon, E)$ such that $q_{t}(\tau, E)=p_{t}\left(\tau^{-1} E\right)$ whenever $\tau$ belongs to $G$. As for $q_{t}(\omega, E)$, it must be 1 or 0 according as $E$ contains $\omega$ or not. Otherwise $q_{t}(\omega, E)$ would be strictly positive for some compact set $E$ not containing $\omega$; there would be a sequence $\sigma_{n}$ such that the sets $\sigma_{n} E$ were pairwise disjoint, and from (6) we should derive the absurd conclusion

$$
1=q_{t}\left(\omega, G_{c}\right) \geqq \sum_{n} q_{t}\left(\omega, \sigma_{n} E\right)=\sum_{n} q_{t}(\omega, E)=\infty .
$$

Since $S_{t}$ and $p_{t}$ clearly stand in the relation (3) to one another, the fact that the $S_{t}$ form a semi-group implies that so do the $p_{t}$. Finally, (2) follows at once from (4).

2.3. Thus there is a one-to-one correspondence between semi-groups of positive measures on $G_{c}$ which satisfy (1) and (2), and semi-groups of linear transformations of $\mathcal{C}$ which satisfy (4), (5) and (6). We shall call $\left(S_{t}\right)$ a positive semi-group and the $p_{t}$ the associated probability measures.

We intend to characterize all positive semi-groups on $G$ by writing down explicitly their infinitesimal generators. In the next section it will be shown that the generator-or rather a corresponding linear functional-must have a certain expression, then in $\$ 4$ that every transformation of this form generates a positive semi-group. The results are summarized in $\$ 5$.

2.4. A finite measure on $G$ must vanish on all but a countable number of cosets of the connected component $\mathfrak{F}$ of $\epsilon$. Thus if the $p_{t}$ are the associated measures of a positive semi-group, the collection $\mathscr{H}$ of those cosets of $\mathcal{H}$ on which $p_{t}$ differs from zero for some rational $t$ is countable. Let $G^{\prime}$ be the separable group generated by the elements of cosets in $\mathcal{X}$ and let $G_{c}^{\prime}$ be the compactification of $G^{\prime}$ using the same point at infinity as for $G$. Now consider 
any function $f$ in $\mathcal{C}$ which vanishes outside some compact set not intersecting $G_{c}^{\prime}$. The integral $\int f(\sigma) p_{t}(d \sigma)$ must vanish for all $t$, because it is continuous in $t$ and vanishes for rational $t$; so for every $t$ the measure $p_{t}$ is confined to $G_{c}^{\prime}$. It is easy to see that the $p_{t}$ define a positive semi-group on $G^{\prime}$ from which the original semi-group on $G$ can be obtained by left translations by elements of $G$.

We may therefore, without loss of generality, restrict our consideration to separable Lie groups whenever this proves convenient.

3. The infinitesimal generator. Let $\left(S_{t}\right)$ be a positive semi-group on $G$ with associated measures $p_{i}$. According to $\$ 1.7$ the domain $\mathcal{D}$ of the infinitesimal generator $M$ is dense in $\mathcal{C}$; we need however more precise information.

3.1. If $f$ belongs to $\mathcal{C}_{1}^{\prime}$ and $Y$ to the Lie algebra of $G$ then

$$
\begin{aligned}
Y^{\prime} S_{t} f & =\lim _{s \rightarrow 0} \frac{1}{s}\left(L_{\text {exp } Y} S_{t} f-S_{t} f\right) \\
& =S_{t} \lim _{s \rightarrow 0} \frac{1}{s}\left(L_{\text {exp } y} Y-f\right) \\
& =S_{t} Y^{\prime} f
\end{aligned}
$$

because $S_{t}$ is a bounded linear transformation which commutes with left translations. Similarly $S_{t}\left(Y^{\prime} Z^{\prime} f\right)=Y^{\prime} Z^{\prime}\left(S_{t} f\right)$ if $f$ belongs to $\mathcal{C}_{2}^{\prime}$, so that $\mathcal{C}_{2}^{\prime}$ is invariant under all $S_{t}$.

It follows from the definition of the norm that $\left\|S_{t} f\right\|_{2}^{\prime} \leqq\|f\|_{2}^{\prime}$ and that $\left\|S_{t} f-f\right\|_{2}^{\prime} \rightarrow 0$ as $t \rightarrow 0$, for every $f$ in $\mathcal{C}_{2}^{\prime}$. Therefore the restrictions of the $S_{t}$ to $\mathcal{C}_{2}^{\prime}$ form a semi-group whose infinitesimal generator is defined, according to $\$ 1.7$, on a dense linear subset $\mathcal{D}^{\prime}$ of $\mathcal{C}_{2}^{\prime}$. Now $\mathscr{D}$ includes $\mathscr{D}^{\prime}$, because convergence in $\mathcal{C}_{2}^{\prime}$ is stricter than convergence in $\mathcal{C}$; so $\mathscr{D} \cap \mathcal{C}_{2}^{\prime}$ is dense in $\mathcal{C}_{2}^{\prime}$.

3.2. Let us set $\zeta_{s}^{i}=\exp s X_{i}$, where $X_{1}, \cdots, X_{d}$ is a basis of the Lie algebra of $G$, and define

$$
\begin{aligned}
D_{i} f & =\lim _{s \rightarrow 0} \frac{1}{s}\left[f\left(\zeta_{s}^{i}\right)-f(\epsilon)\right], \\
D_{i j} f & =\lim _{s \rightarrow 0} \frac{1}{s^{2}}\left[f\left(\zeta_{s}^{i} \zeta_{s}^{j}\right)-f\left(\zeta_{s}^{i}\right)-f\left(\zeta_{s}^{j}\right)+f(\epsilon)\right]
\end{aligned}
$$

for every function $f$ which is twice differentiable in a neighborhood of $\epsilon$. Note that $D_{i} f$ is $X_{i} f \cdot(\epsilon)$ and $D_{i j} f$ is $X_{i} X_{j} f \cdot(\epsilon)$ if $f$ belongs to $\mathcal{C}_{2}$, whereas $D_{i} f$ is $-X_{i}^{\prime} f \cdot(\epsilon)$ and $D_{i j} f$ is $X_{j}^{\prime} X_{i}^{\prime} f \cdot(\epsilon)$ if $f$ belongs to $\mathcal{C}_{2}^{\prime}$. The restriction of $D_{i}$ or $D_{i j}$ to $\mathcal{C}_{2}$ or $\mathcal{C}_{2}^{\prime}$ is a continuous linear functional.

The following lemma is a consequence of the preceding number and of the elementary geometry of Banach spaces.

Lemma. For every $f$ in $\mathcal{C}_{2}^{\prime}$ and every positive $\delta$ there is a $g$ in $\Phi \cap \mathcal{C}_{2}^{\prime}$ such that $\|f-g\|_{2}^{\prime}<\delta, D_{i} g=D_{i} f, D_{i j} g=D_{i j} f$. 
An appropriate use of this lemma yields the existence of a function $\phi$ in $\mathcal{D} \cap \mathcal{C}_{2}^{\prime}$ which satisfies

$$
\phi(\epsilon)=D_{i} \phi=0, \quad D_{i i} \phi=2, \quad D_{i j} \phi=0 \text { if } i \neq j,
$$

and which is strictly positive on $G_{c}-\{\epsilon\}$. In coordinates $x_{i}$ adapted to the basis $X_{i}$ at $\epsilon$ - that is to say, $x_{i}(\epsilon)=0$ and $D_{i} x_{j}=\delta_{i j}$ - the function $\phi$ behaves like $\sum x_{i}^{2}$ near $\epsilon$.

3.3. Let $\varepsilon$ be the class of functions which belong to $\mathcal{C}$ and are twice continuously differentiable on $G$. Once the function $\phi$ has been fixed, it follows easily from the lemma that for every $f$ in $\varepsilon$ and every positive $\delta$ there is a $g$ in $\mathscr{D} \cap \mathcal{C}_{2}^{\prime}$ such that

$$
|f(\tau)-g(\tau)| \leqq \delta \phi(\tau), \quad \tau \in G_{c} .
$$

This being so, we prove that the limit

$$
\begin{aligned}
A f & =\lim _{\lambda 00} \frac{1}{t}\left[S_{t} f \cdot(\epsilon)-f(\epsilon)\right] \\
& =\lim _{t \searrow 0} \frac{1}{t} \int_{G_{c}}[f(\sigma)-f(\epsilon)] p_{t}(d \sigma)
\end{aligned}
$$

exists for every $f$ in $\mathcal{E}$. Given $\delta>0$ take $g$ in $\mathcal{D} \cap \mathcal{C}_{2}^{\prime}$ to satisfy (1). Then

$$
\frac{1}{t} \int|f(\sigma)-g(\sigma)| p_{t}(d \sigma) \leqq \frac{\delta}{t} \int \phi(\sigma) p_{t}(d \sigma)
$$

and the right member is $O(\delta)$ because $\phi$ is in $\mathcal{D}$. Consequently the functions $t^{-1} \int[f(\sigma)-f(\epsilon)] p_{t}(d \sigma)$ and $t^{-1} \int[g(\sigma)-g(\epsilon)] p_{t}(d \sigma)$ differ by $O(\delta)$; since the latter function has a limit as $t$ decreases to 0 (because $g$ is in $\mathcal{D}$ ) and since $\delta$ is arbitrary, the limit in (2) must exist.

3.4. Consider the family of positive measures

$$
F_{t}(E)=\frac{1}{t} \int_{E} \phi(\sigma) p_{t}(d \sigma), \quad E \subset G_{c} .
$$

Because $t^{-1} \int \phi p_{t}(d \sigma)$ has a limit the family is uniformly bounded in $t$, and according to relation (2) the integral

$$
\int_{G^{-}-\{\in\}} f(\sigma) F_{t}(d \sigma)=\frac{1}{t} \int_{G_{c}} f(\sigma) \phi(\sigma) p_{t}(d \sigma)
$$

converges, as $t$ decreases to 0 , for every $f$ in $\varepsilon$. Hence the $F_{t}$ converge weakly on $G_{c}-\{\epsilon\}$ to a finite positive measure $F$ and consequently

$$
\frac{1}{t} \int_{G_{c}} f(\sigma) \phi(\sigma) p_{t}(d \sigma) \rightarrow \int_{G_{c-\{\epsilon\}}} f(\sigma) F(d \sigma)
$$


for every function $f$ belonging to $\mathcal{C}$ and vanishing at $\epsilon$.

We make use again of the lemma in $\$ 3.2$ to determine elements $x_{i}$ and $x_{i j}$ of $\mathcal{D} \cap \mathcal{C}_{2}^{\prime}$ such that

$$
\begin{aligned}
& x_{i}(\epsilon)= x_{i j}(\epsilon)=D_{i} x_{j k}=0, \quad D_{i} x_{j}=\delta_{i j}, \\
& D_{i j} x_{k_{l}}= \begin{cases}0, & \{i, j\} \neq\{k, l\}, \\
1, & \{i, j\}=\{k, l\}, i \neq j, \\
2, & i=j=k=l .\end{cases}
\end{aligned}
$$

Thus the $x_{i}$ serve as coordinates near $\epsilon$ and the $x_{i j}$ behave like the products $x_{i} x_{j}$ through terms of the second order at $\epsilon$. We set $b_{i}=A x_{i}$ and $b_{i j}=A x_{i j}$ by way of abbreviation.

Take any $f$ in $\varepsilon$ and set $c=f(\epsilon), c_{i}=D_{i} f, 2 c_{i j}=D_{i j}\left(f-\sum c_{k} x_{k}\right)$. Then the function $f-c-\sum c_{i} x_{i}-\sum c_{i j} x_{i j}$ belongs to $\varepsilon$ and has the form $h \phi$ with $h$ belonging to $\mathcal{C}$ and vanishing at $\epsilon$. Therefore

$$
\begin{aligned}
A f & =A\left(c+\sum c_{i} x_{i}+\sum c_{i j} x_{i j}\right)+\lim _{t \searrow 0} \frac{1}{t} \int_{G_{c}} h \phi p_{t}(d \sigma) \\
& =\sum b_{i} c_{i}+\sum b_{i j} c_{i j}+\int_{G^{c-\{\epsilon\}}}\left(f-c-\sum c_{i} x_{i}-\sum c_{i j} x_{i j}\right) \phi^{-1} F(d \sigma)
\end{aligned}
$$

where $h$ has been written out explicitly in the last integral.

Each integral $\int x_{i j} \phi^{-1} F(d \sigma)$ makes sense, because $x_{i j} / \phi$ is bounded and continuous on $G_{c}-\{\epsilon\}$. So we may drop the terms $\int c_{i j} x_{i j} \phi^{-1} F(d \sigma)$ from the last integral in (3) if we agree to adsorb them into the sum $\sum b_{i j} c_{i j}$ by changing the values of the $b_{i j}$ appropriately. After making this change we write

(4) $A f=\sum a_{i} D_{i} f+\sum a_{i j} D_{i j} f+\int_{G^{c^{-}\{\epsilon\}}}\left[f(\sigma)-f(\epsilon)-\sum\left(D_{i} f\right) x_{i}(\sigma)\right] G(d \sigma)$

where $G$ is the positive measure $G(d \sigma)=\phi^{-1}(\sigma) F(d \sigma)$ on $G_{c}-\{\epsilon\}$. The coefficients $a_{i j}$ we take to be symmetric in the indices, for $D_{i j}-D_{j i}$ is a linear combination of the $D_{k}$.

3.5. It is easy to verify that the matrix $\left(a_{i j}\right)$ is positive semi-definite by considering those $f$ in $\varepsilon$ for which $f(\tau) \geqq 0$ for all $\tau$ and $f(\epsilon)=D_{i} f=0$. Since $S_{t} f$ is positive,

$$
\sum a_{i j} D_{i j} f+\int_{G^{c-\{\epsilon\}}} f(\sigma) G(d \sigma)=\lim _{\lambda_{0}} t^{-1} S_{t} f \cdot(\epsilon) \geqq 0 .
$$

Assume, for the purpose of arguing by reductio ad absurdum, that $\sum a_{i j} D_{i j} f$ is strictly negative for some $f$. Take a sequence of functions, $f_{n}=f \exp \left(-n \phi^{2}\right)$ say, which satisfy the same conditions as $f$ and are such that $D_{i j} f_{n}=D_{i j} f$ and $\int f_{n} G(d \sigma) \rightarrow 0$; then the left member of (5), written with $f_{n}$ replacing $f$, is 
less than 0 for large $n$. This is a contradiction; consequently $\sum a_{i j} D_{i j} f$ is never negative. Now, as $f$ varies the matrix $\left(D_{i j} f\right)$ ranges over all symmetric positive semi-definite matrices; since $\sum a_{i j} D_{i j} f$ remains non-negative, the matrix $\left(a_{i j}\right)$ must be positive semi-definite.

3.6. It is clear that $M f \cdot\left(\tau^{-1}\right)=A\left(L_{\tau} f\right)$ whenever $M f$ is defined and $\tau$ belongs to $G$, so that the expression (4) of $A$ can be translated into one for $M f \cdot(\tau)$ provided $f$ belongs to $\mathscr{D} \cap \mathcal{E}$. Although it would not be difficult to prove at this point that $\mathcal{D}$ includes $\mathcal{C}_{2}$, and thus to obtain the expression of $M$ restricted to $\mathcal{C}_{2}$, we prefer to wait until $\S 4$. There, however, we shall need to know that the restriction of the linear functional $A$ to $\mathcal{C}_{2}$ determines the semigroup $\left(S_{t}\right)$. We proceed to the proof of this statement.

Once the $x_{i}$ have been chosen, $A$ on $\mathcal{C}_{2}$ determines the constants $a_{i}, a_{i j}$ and the measure $G$; so $A$ on $\mathcal{C}_{2}$ determines $A$ on $\varepsilon$. Now consider any function $f$ in $\varepsilon_{\text {which }}$ is constant near $\omega$; since such functions are dense in $\mathcal{C}$ it suffices to prove that $A$ on $\varepsilon$ determines $S_{t} f$ for all $t$. We have already observed that $S_{t} f \cdot(\omega)$ has the constant value $f(\omega)$. Moreover, $S_{t} f$ belongs to $\varepsilon$ because $f$ belongs to $\mathcal{C}_{2}^{\prime}$ and $S_{t}$ transforms $\mathcal{C}_{2}^{\prime}$ into itself. Hence, setting $f_{0}(\tau)=f(\tau)$ and $f_{t}(\tau)=S_{t} f \cdot(\tau)$, we obtain a continuous function on $[0, \infty) \times G_{c}$ which satisfies the equations

$$
\begin{array}{cr}
\frac{\partial f_{t}(\omega)}{\partial t}=0, & t>0, \\
\frac{\partial f_{t}(\tau)}{\partial t}=\lim _{\delta>0} \frac{1}{s}\left[S_{s} f_{t} \cdot(\tau)-f_{t}(\tau)\right]=A\left(L_{\tau^{-1}} f_{t}\right), & \tau \in G, t>0,
\end{array}
$$

where $f_{t}$ stands for $f_{t}(\sigma)$ considered as a function of $\sigma$. It suffices to compute the derivative with respect to $t$ on the right as we have done, for the resulting expression is continuous in $t$ for fixed $\tau$ since $A \circ L_{\tau^{-1}}$ is a continuous linear functional and $\left(S_{t}\right)$ a strongly continuous semi-group on $\mathcal{C}_{2}^{\prime}$.

The fact that $A$ on $\mathcal{C}_{2}$ determines the $S_{t}$ is therefore equivalent to the statement that the system (6) has a unique solution once $f_{0}$ is prescribedthat is to say, to the statement: Let $g_{t}(\tau)$ be a continuous function on the space $[0, \infty) \times G_{c}$ such that $g_{0}(\tau)=0$ for all $\tau, g_{t}(\omega)=0$ for all $t, \partial g_{t}(\tau) / \partial t$ exists for all $\tau$ and all positive $t$, and $g_{t}(\tau)$ is twice differentiable on $G$ for each $t$; if also

$$
\frac{\partial g_{t}(\tau)}{\partial t}=A\left(L_{\tau^{-1}} g_{t}\right), \quad \tau \in G, t>0,
$$

then $g_{t}(\tau)$ vanishes identically.

This last statement is an instance of the assertion in the first paragraph of $\$ 1.8$ (not of the lemma there), for $A L_{\tau^{-1}} g_{t}$ is non-negative if $g_{t}$ takes on its minimum at $\tau$.

4. The generation of positive semi-groups. The preceding section showed that a positive semi-group defines on $\mathcal{C}_{2}$ a linear functional which determines 
the semi-group. Turning matters around, we prove that every linear functional satisfying the necessary conditions gives rise to a positive semi-group. The argument depends on the following lemma.

4.1. Lemma. Let $\left(S_{t}^{k}\right)$ be a sequence of positive semi-groups whose infinitesimal generators $M^{k}$ are defined at least on $\mathcal{C}_{2}$. Suppose moreover that for each $f$ in $\mathcal{C}_{2}$ the sequence $M^{k} f$ converges, say to $M^{\prime} f$, in the metric of $\mathcal{C}$. Then $S_{t}^{k} f$ converges, say to $S_{t} f$, for every $f$ in $\mathcal{C}$ and every positive $t$, and the transformations $S_{t}$ form a positive semi-group whose infinitesimal generator is defined at least on $\mathcal{C}_{2}$ and coincides there with $M^{\prime}$.

For $f$ in $\mathcal{C}_{2}$ we have

$$
\left\|\frac{d}{d t} S_{t}^{k} f\right\|=\left\|S_{t}^{k} M^{k} f\right\| \leqq\left\|M^{k} f\right\|
$$

so that the $S_{t}^{k} f$, considered as functions of $t$, have a common modulus of uniform continuity.

A subsequence of the associated measures $p_{i}^{k}$ converges weakly for every rational $t$. In order not to complicate the notation we assume until the end of the argument that the full original sequence so converges.

The inequality above implies that for $f$ in $\mathcal{C}_{2}$ and $\tau$ in $G_{c}$ the functions $S_{t}^{k} f \cdot(\tau)$ of $t$ have a common modulus of continuity independent of $k$; so the sequence $\left(p_{t}^{k}\right)$ converges weakly for all $t$, say to $p_{t}$. Clearly each $p_{t}$ is positive and $p_{t}\left(G_{c}\right)=1$. Let $S_{t}$ be the transformation $S_{t} f \cdot(\tau)=\int f(\tau \sigma) p_{t}(d \sigma)$ of $\mathcal{C}$ into itself, so that $S_{t}^{k} f$ converges weakly to $S_{t} f$ for every $f$ in $\mathcal{C}$. We obtain

$$
S_{t} f-f=\int_{0}^{t} S_{s} M^{\prime} f d s, \quad f \in \mathcal{C}_{2},
$$

with a weak integral by writing the same equation for the pair $S_{t}^{k}, M^{k}$ and then passing to the limit. This equation implies that

$$
\left\|S_{t+h} f-S_{t} f\right\| \leqq \int_{t}^{t+h}\left\|S_{\imath} M^{\prime} f\right\| d s \leqq\left\|M^{\prime} f\right\| h, \quad f \in \mathcal{C}_{2},
$$

so that $S_{t} f$ is strongly continuous for each $f$ in $\mathcal{C}_{2}$-hence also for each $f$ in $\mathcal{C}$, because $\mathcal{C}_{2}$ is dense in $\mathcal{C}$ and $S_{t}$ has bound 1. Equation (1) shows too that $S_{t} f$ converges strongly to $f$ as $t \rightarrow 0$, first for $f$ in $\mathcal{C}_{2}$ and then for $f$ in $\mathcal{C}$. We may now interpret the integral in (1) as a strong integral and obtain

$$
\begin{aligned}
\lim _{t>0} \frac{1}{t}\left(S_{t} f-f\right) & =\lim _{\Delta, 0} \frac{1}{t} \int_{0}^{t} S_{s} M^{\prime} f d s & \\
& =M^{\prime} f, & f \in \mathcal{C}_{2},
\end{aligned}
$$

the limit being in the metric of $\mathcal{C}$. 
The last relation enables us to prove that $p_{t}^{k}(G)$ tends to $p_{t}(G)$. Since $p_{t}(G) \leqq \lim \inf p_{t}^{k}(G)$ by the definition of weak convergence, it suffices to prove that $p_{t}(G) \geqq \lim \sup p_{t}^{k}(G)$. From $\S 3$ we have the representation

$$
M^{k} f \cdot(\epsilon)=\Delta^{k} f \cdot(\epsilon)+\int_{G^{-}-\{\epsilon\}}\left[f(\sigma)-f(\epsilon)-\sum X_{i} f \cdot(\epsilon) x_{i}(\sigma)\right] G^{k}(d \sigma)
$$

for $f$ in $\mathcal{C}_{2}$. Each $M^{k}$ is a bounded transformation from the Banach space $\mathcal{C}_{2}$ to the Banach space $\mathcal{C}$ and the $M^{k}$ converge strongly to $M^{\prime}$. It follows easily that $M^{\prime}$ too has the form

$$
M^{\prime} f \cdot(\epsilon)=\Delta f \cdot(\epsilon)+\int_{G^{c-\{\epsilon\}}}\left[f(\sigma)-f(\epsilon)-\sum X_{i} f \cdot(\epsilon)\right] G(d \sigma)
$$

with $\Delta$ and $G$ satisfying the conditions derived in $\$ 3$, and that in particular $G^{k}(\omega) \rightarrow G(\omega)$.

We first suppose that $G(\omega)=0$. Let $A$ be a compact neighborhood of $\epsilon$ in $G$ and take $f$ in $\mathcal{C}_{2}$ so that $0 \leqq f(\tau) \leqq 1$ for all $\tau, f(\tau)=1$ for $\tau$ in $A$, and $f(\omega)=0$. Equation (2) implies

$$
\begin{aligned}
\liminf _{\lambda 0} \frac{1}{t}\left[p_{t}(G)-1\right] & \geqq \lim _{\searrow_{0}} \frac{1}{t}\left[S_{t} f \cdot(\epsilon)-1\right] \\
& =M^{\prime} f \cdot(\epsilon) \\
& =\int_{G^{-A}}[f(\sigma)-1] G(d \sigma) \\
& \geqq-G(G-A) .
\end{aligned}
$$

Since $G(G-A)$ can be made arbitrarily small it follows that

$$
\lim _{\supset 0} \frac{1}{t}\left[1-p_{t}(G)\right]=0
$$

Let us fix $s$ and write $p_{s}(G)=e^{-c s}$ with $c \geqq 0$. Let $B$ be any compact set in $G$ and $C$ the set of products $\sigma \tau$ with both factors in $B$. The definition of convolution shows that $\left[p_{s / 2}^{k}(B)\right]^{2} \leqq p_{s}^{k}(C)$ for each $k$. Consequently

$$
\begin{aligned}
p_{s / 2}(G) & =\sup _{B} \limsup _{k \rightarrow \infty} p_{s / 2}^{k}(B) \\
& \leqq \sup _{B} \limsup _{k \rightarrow \infty}\left[p_{s}^{k}(C)\right]^{1 / 2} \\
& =e^{-c s / 2} .
\end{aligned}
$$

Repetition of this argument gives the inequality $p_{s / 2^{n}}(G) \leqq e^{-c s / 2^{n}}$, which in turn implies 


$$
\liminf _{t>0} \frac{1}{t}\left[p_{t}(G)-1\right] \leqq-c
$$

On comparing this result with the former equality we find that $c$ must vanish. Thus $p_{s}(G)=1$ and consequently $p_{s}^{k}(G) \rightarrow p_{s}(G)$ for every $s$.

When $G(\omega)$ differs from 0 the proof is a little more complicated. Using the fact that $G^{k}(\omega) \rightarrow G(\omega)$ and that $p_{t}^{k}(G)=\exp \left\{-G^{k}(\omega) t\right\}$ one throws the proof back upon the case we have dealt with. We omit the details.

From the convergence of $p_{t}^{k}(G)$ to $p_{t}(G)$ follows the strong convergence of $S_{t}^{t}$ to $S_{t}$. Since the bound of each $S_{t}$ is 1 we now obtain the equation $S_{s} S_{t}=S_{s+t}$ by passing to the limit in the equation $S_{s}^{k} S_{t}^{k}=S_{s+1}^{k}$. Therefore the family $\left(S_{t}\right)$ is a positive semi-group; and by (2) the restriction of the generator of $\left(S_{t}\right)$ to $C_{2}$ is precisely $M^{\prime}$.

We have proved the lemma with the original sequence replaced by a subsequence. But note that $M^{\prime}$, according to $\$ 3.6$, determines the limit semigroup $\left(S_{t}\right)$. Thus our construction is quite independent of the particular subsequence which may have been chosen. This being so, a familiar argument can now be used to prove that the full original sequence of semi-groups must converge strongly to the semi-group $\left(S_{t}\right)$. This completes the proof of the lemma.

4.2. Let $x_{1}, \cdots, x_{d}$ be functions in $\bigodot_{2}$ such that $x_{i}(\epsilon)=0$ and $X_{i} x_{j} \cdot(\epsilon)$ $=D_{i} x_{j}=\delta_{i j}$, and let $\phi$ be a function in $\mathcal{C}_{2}$ which is strictly positive on $G_{c}-\{\epsilon\}$ and behaves near $\epsilon$ like $\sum x_{i}^{2}$ through terms of the second order. (The $x_{i}$ and $\phi$ occurring in $\$ 3$ were required to be in $\mathcal{D} \cap \mathcal{C}_{2}^{\prime}$; once formula (4) of $\S 3$ has been proved, however, that restriction may be replaced by the present one.)

Let $N$ be defined on $C_{2}$ by the equation

$$
\begin{aligned}
N f \cdot(\tau)= & \sum a_{i} X_{i} f \cdot(\tau)+\sum a_{i j} X_{i} X_{j} f \cdot(\tau) \\
& +\int_{G^{-\{\epsilon\}}}\left[f(\tau \sigma)-f(\tau)-\sum X_{i} f \cdot(\tau) x_{i}(\sigma)\right] G(d \sigma)
\end{aligned}
$$

where $\left(a_{i j}\right)$ is a symmetric positive semi-definite matrix and $G$ is a positive measure on $G_{c}-\{\epsilon\}$ for which the integral $\int \phi G(d \sigma)$ is finite. Note that $N f \cdot(\omega)=0$. It is clear from the definition of $\mathcal{C}_{2}$ that $N$ is a bounded linear transformation from the Banach space $C_{2}$ to the Banach space $\mathcal{C}$. If $G$ and the $x_{i}$ are the same in (3) and in equation (4) of $\S 3$, then $N f \cdot(\tau)=A\left(L_{\tau}^{-1} f\right)$ for $\tau$ in $G$.

We shall prove that $N$ is the restriction to $C_{2}$ of the infinitesimal generator of exactly one positive semi-group.

4.3. First consider an $N$ which can be written

$$
N f \cdot(\tau)=\int_{G^{c}-\{\epsilon\}}[f(\tau \sigma)-f(\tau)] F(d \sigma), \quad \tau \in G_{c}
$$


with $F$ a finite measure on $G_{c}-\{\epsilon\}$; such an $N$ is obviously an instance of (3). Equation (4) defines $N$ as a linear transformation from $\mathcal{C}_{2}$ to $\mathcal{C}$ whose bound is twice the total measure of $F$ when $\mathcal{C}_{2}$ is considered a submanifold of $\mathcal{C}$. Consequently $N$ extends by continuity to a linear transformation $N^{\prime}$ of $\mathcal{C}$ into itself with the same bound as before and the same expression. It follows that the transformations $S_{t} \equiv \sum\left(t N^{\prime}\right)^{n} / n$ ! for $t \geqq 0$ form a semi-group of transformations of $\mathcal{C}$ which is continuous in the uniform topology of operators. Since $N f \cdot(\tau)$ is non-negative whenever the minimum of $f$ occurs at $\tau$, the lemma of $\$ 1.8$ implies that equation (5) of $\S 2$ holds. Finally, $S_{t}$ commutes with left translations because $N^{\prime}$ does so.

Thus $N$ is the restriction to $\mathcal{C}_{2}$ of the infinitesimal generator of a positive semi-group.

4.4. We settle the general case by approximation, using the result in $\S 1.6$ and the lemma in $\$ 4.1$. If

$$
N f \cdot(\tau)=\sum a_{i} X_{i} f \cdot(\tau)+\int_{G^{c^{-}(e)}}\left[f(\tau \sigma)-f(\tau)-\sum X_{i} f \cdot(\tau) x_{i}(\sigma)\right] F(d \sigma)
$$

with $F$ a finite positive measure, we may write

$$
N f \cdot(\tau)=Y f \cdot(\tau)+\int_{G^{(-\{\epsilon\}}}[f(\tau \sigma)-f(\tau)] F(d \sigma),
$$

with $Y$ in the Lie algebra of $G$. There is a sequence of finite positive measures $\mu_{n}$ on $G_{c}-\{\epsilon\}$ such that $\int[f(\tau \sigma)-f(\tau)] \mu_{n}(d \sigma)$ tends to $Y f$ in the metric of $\mathcal{C}$ for every $f$ in $\mathcal{C}_{2}$, and by $\$ 4.3$ the transformations

$$
N_{n} f \cdot(\tau)=\int_{G^{c-\{\epsilon\}}}[f(\tau \sigma)-f(\tau)]\left[G(d \sigma)+\mu_{n}(d \sigma)\right], \quad f \in \mathcal{C},
$$

are the generators of positive semi-groups. So the lemma ensures that $N$ also is the restriction to $\mathcal{C}_{2}$ of the infinitesimal generator of a positive semi-group.

Consider finally the general case of (3). Take a sequence of finite positive measures $\mu_{n}$ on $G_{c}-\{\epsilon\}$ so that $\int[f(\tau \sigma)-f(\tau)] \mu_{n}$ tends in $\mathcal{C}$ to $\sum a_{i j} X_{i} X_{j} f$ for every $f$ in $\mathcal{C}_{2}$, and let $G_{n}$ be the finite measure $\left(1-e^{-n \phi}\right) G(d \sigma)$ on $G_{c}-\{\epsilon\}$. If we define

$$
\begin{aligned}
N_{n} f \cdot(\tau)= & \sum a_{i} X_{i} f \cdot(\tau)+\int_{G_{c-\{\epsilon\}}}[f(\tau \sigma)-f(\tau)] \mu_{n}(\dot{d \sigma}) \\
& +\int_{G^{-\{}\{\epsilon\}}\left[f(\tau \sigma)-f(\tau)-\sum X_{i} f \cdot(\tau) x_{i}(\sigma)\right] G_{n}(d \sigma)
\end{aligned}
$$

then $N_{n}$ coincides on $\mathcal{C}_{2}$ with the infinitesimal generator of a positive semigroup and also $N_{n} f \rightarrow N f$ in the metric of $\mathcal{C}$ for every $f$ in $\mathcal{C}_{2}$. Hence $N$ itself is 
the restriction to $\mathcal{C}_{2}$ of the infinitesimal generator of a positive semi-group.

4.5. At this point we verify that the infinitesimal generator of a positive semi-group is always defined on $\mathcal{C}_{2}$. Let $\left(S_{t}\right)$ be the semi-group and $A$ the linear functional defined in $\S 3.3$ but restricted now to $\mathcal{C}_{2}$. We take the $a_{i}, a_{i j}$, and $G$ in (3) so that $N f \cdot(\boldsymbol{\epsilon})=A f$. Now, $N$ is the restriction to $\mathcal{C}_{2}$ of the infinitesimal generator of some positive semi-group, say $\left(S_{t}^{\prime}\right)$. But, according to $\S 3.6$, the two semi-groups $\left(S_{t}\right)$ and $\left(S_{t}^{\prime}\right)$ must coincide, for they both induce the linear functional $A$ on $\mathrm{C}_{2}$.

This argument also proves that the $N$ of (3) is the restriction to $\mathcal{C}_{2}$ of the infinitesimal generator of only one probability semi-group.

5. Statement of results. As usual $x_{1}, \cdots, x_{d}$ are functions in $\mathcal{C}_{2}$ for which $x_{i}(\epsilon)=0$ and $X_{i} x_{j} \cdot(\epsilon)=\delta_{i j}$, and $\phi$ is a function in $\mathcal{C}_{2}$ which is strictly positive on $G_{c}-\{\epsilon\}$ and behaves near $\epsilon$ like $\sum x_{i}^{2}$.

5.1. Theorem. Let $\left(S_{t}\right)$ be a positive semi-group on the Lie group $G$. Its infinitesimal generator $M$ is defined at least on $\mathcal{C}_{2}$ and has there the representation

$$
\begin{aligned}
M f \cdot(\tau)= & \sum a_{i} X_{i} f \cdot(\tau)+\sum a_{i j} X_{i} X_{j} f \cdot(\tau) \\
& +\int_{G_{c-\mid \epsilon\}}}\left[f(\tau \sigma)-f(\tau)-\sum X_{i} f \cdot(\tau) x_{i}(\sigma)\right] G(d \sigma) .
\end{aligned}
$$

Here the matrix $\left(a_{i j}\right)$ is symmetric positive semi-definite and $G$ is a positive measure on $G_{c}-\{\epsilon\}$ for which the integral $\int \phi(\sigma) G(d \sigma)$ is finite. The measure $G$ and the operator $\Delta_{2} \equiv \sum a_{i j} X_{i} X_{j}$ are determined by $M$ independently of the choice of the basis $X_{1}, \cdots, X_{d}$ and the functions $x_{i}$. Moreover, the restriction of $M$ to $\mathcal{C}_{2}$ determines the semi-group $\left(S_{t}\right)$.

Conversely, if the transformation $M: \mathcal{C}_{2} \rightarrow \mathcal{C}$ is defined by (1) and if the matrix $\left(a_{i j}\right)$ and the measure $G$ satisfy the conditions above, then $M$ is the restriction to $\mathcal{C}_{2}$ of the infinitesimal generator of exactly one probability semi-group.

We have proved all the statements of the theorem except the uniqueness of $\Delta_{2}$ and $G$. To see that $G$ is unique we need only observe that $M f \cdot(\epsilon)$ is just $\int f(\sigma) G(d \sigma)$ if the function $f$ vanishes near $\epsilon$. This being so, the part of $M$ not accounted for by the integral is determined once the $X_{i}$ and $x_{i}$ have been fixed. But changing the $x_{i}$ changes only the first order operator $\Delta_{1} \equiv \sum a_{i} X_{i}$, so that $\Delta_{2}$ depends at most on the $X_{i}$. In performing a substitution on the $X_{i}$ we may subject the $x_{i}$ to the contragredient substitution; the integral then remains invariant, so also $\Delta_{1}+\Delta_{2}$, and the discussion in $\$ 1.5$ shows that $\Delta_{2}$ does not depend on the choice of the $X_{i}$.

The term $\Delta_{1}$ depends of course on the choice of the functions $x_{i}$. For certain groups - for example, compact semi-simple Lie groups-or for positive semi-groups with certain properties of symmetry, one can choose the $x_{i}$ "naturally" for each basis $X_{1}, \cdots, X_{d}$ so that $\Delta_{1}$ becomes uniquely determined. 
5.2. We add a few remarks whose proofs are easy.

First, $G(\omega)=0$ if $p_{t}(\omega)=0$ for some $t$; conversely $p_{t}(\omega)=0$ for all $t$ if $G(\omega)=0$.

Second, $M$ is a bounded operator on $\mathcal{C}$ if and only if

$$
M f \cdot(\tau)=\int_{G^{c-[\epsilon]}}[f(\tau \sigma)-f(\tau)] F(d \sigma), \quad \tau \in G_{c},
$$

with $F$ a finite positive measure on $G_{c}-\{\epsilon\}$. If $G$ is discrete then $M$ necessarily has this form.

Third, the set of generators of positive semi-groups on $G$ is a convex cone in which operators of the form (2) are dense. To be precise, let $\mathcal{X}($ be the set of restrictions to $\mathcal{C}_{2}$ of the infinitesimal generators of all positive semigroups on $G$. Then $\mathcal{X}$ is a closed subset in the space of continuous linear transformations from the Banach space $\mathcal{C}_{2}$ to the Banach space $\mathcal{C}$ (in the strong topology of operators); $\mathcal{X}$ is closed under the operation of forming linear combinations with positive coefficients; and transformations of the form (2) are dense in $\mathcal{X}$ (see the argument in $\S 4.1$ ). It can even be shown that the $M$ of the form $\sum c_{i}\left(R_{\sigma_{i}}-1\right)$ with positive $c_{i}$ are dense in $\mathcal{X}$.

5.3. We sketch the probability interpretation of our results under the hypothesis that $p_{t}(\omega)=0$. The definition $q_{t}(\tau, E)=p_{t}\left(\tau^{-1} E\right)$ yields stationary Markoff transition probabilities on $G$ which are invariant under left translations: $q_{t}(\sigma \tau, \sigma E)=q_{t}(\tau, E)$. Conversely, left invariant transition probabilities $q_{t}(\tau, E)$ define a semi-group of measures $p_{t}(E)=q_{t}(\epsilon, E)$ which satisfy $(1)$ and (2) of $\S 2$ provided $q_{t}(\tau, E) \rightarrow 1$ as $t \rightarrow 0$ whenever $E$ is a neighborhood of $\tau$. On an appropriate probability space one can realize the transition probabilities by a stationary process of random variables $\zeta(t)$ with values in $G$ and independent increments. This is to say, $\operatorname{Pr}\left\{\zeta(s)^{-1} \zeta(t) \in E\right\}=p_{t-8}(E)$ for $0 \leqq s<t$ and the increments $\zeta(0)^{-1} \zeta\left(t_{1}\right), \cdots, \zeta\left(t_{n-1}\right)^{-1} \zeta\left(t_{n}\right)$ are independent if the $t_{k}$ are increasing.

When $M=c R_{\sigma}-c$ with positive $c$ and $\zeta(0)=\epsilon$, the $\zeta(t)$ form a Poisson process in the following sense. If the cyclic group $\left\{\sigma^{k}\right\}$ is infinite then

$$
S_{t} f \cdot(\tau)=e^{-c t} \sum_{k \geqq 0} \frac{(c t)^{k}}{k !} f\left(\tau \sigma^{k}\right)
$$

and $\zeta(t)$ takes on the value $\sigma^{k}$ with probability $e^{-c t}(c t)^{k} / k$ ! for non-negative $k$. If $\left\{\sigma^{k}\right\}$ has finite order $r$ then

$$
S_{t} f \cdot(\tau)=\sum_{0 \leqq k<r} P_{k}(c t) f\left(\tau \sigma^{k}\right)
$$

with $P_{k}(u)=e^{-u} \sum u^{n} / n$ !, the sum being extended over the non-negative $n$ congruent to $k$ modulo $r$.

If $M=\Delta_{1}$ then $\zeta(t)=\exp t \Delta_{1}$ and $S_{t}$ is just right translation by the group element. The processes for which $M=\Delta_{1}+\Delta_{2}$, the Brownian motions in the 
group space, have been treated in detail by K. Ito [2]. He has proved that $\zeta(t)$ can be constructed so that each sample path is continuous in $t$; it is not difficult to show that this is possible only when $M=\Delta_{1}+\Delta_{2}$.

\section{Complements}

6. The Lévy formula. Theorem 5.1 as it stands does not include the formula of Lévy, for the characters $e^{i \xi \sigma}$ do not belong to $\mathcal{C}_{2}$. We proceed to strengthen the theorem under the hypothesis that $p_{t}(\omega) \equiv 0$.

6.1. The group $G$.was compactified in order to achieve a perfect reciprocity between measures and certain transformations, and to catch masses escaping to infinity. If we consider only measures confined to $G$ it is better to dispense with $\omega$ altogether and to replace $\mathcal{C}$ by $\overline{\mathcal{C}}$, the space of functions $f$ which are bounded on $G$ and are uniformly continuous in the left uniform structure on $G$, that is to say, $f(\tau \sigma)-f(\tau)$ is small for all $\tau$ if $\sigma$ is sufficiently close to $\epsilon$. It is a little more convenient later on to define $\overline{\mathcal{C}}$ in the following way: Choose a left invariant Riemannian metric on $G$ and take $\overline{\mathcal{C}}$ to be the space of functions which are bounded and uniformly continuous in this metric.

$\overline{\mathcal{C}}$ is a Banach space with the norm $\|f\|=\sup |f(\tau)|$; it includes $\mathcal{C}$ in an obvious fashion. Clearly left translations leave $\overline{\mathcal{C}}$ invariant. To see that so do right translations, express $R_{\sigma} f \cdot(\tau \zeta)-R_{\sigma} f \cdot(\tau)$ as $f\left(\tau \sigma \cdot \sigma^{-1} \zeta \sigma\right)-f(\tau \sigma)$ and note that every neighborhood of $\epsilon$ includes a neighborhood of the form $\sigma^{-1} V \sigma$. Indeed, one neighborhood $V$ will serve for all $\sigma$ in a given compact set; thus as $\sigma$ ranges over a compact set the functions $R_{\sigma} f$ have a common modulus of uniform continuity. It follows that $g(\tau) \equiv \int f(\tau \sigma) \mu(d \sigma)$ lies in $\overline{\mathcal{C}}$ if $f$ does so and $\mu$ is a finite measure. For the integral may be split into two, one over a compact set $E$ and the other over $G-E$; the first is uniformly continuous by the remark just made, the second is uniformly small if $\mu(G-E)$ is small.

The spaces $\overline{\mathcal{C}}_{k}$ are defined as in $\S 1.3$. Each element of the Lie algebra of $G$ defines a continuous transformation of $\overline{\mathcal{C}}_{k}$ into $\overline{\mathcal{C}}_{k-1}$ by the first equation of $\$ 1.3$.

6.2. If $\left(p_{t}\right)$ is a semi-group of positive measures on $G$, so that $p_{t}(\omega) \equiv 0$, and if (1) and (2) of $\$ 2$ hold, then the equations $\bar{S}_{t} f \cdot(\tau)=\int f(\tau \sigma) p_{t}(d \sigma)$ define a semi-group $\left(\bar{S}_{t}\right)$ on $\overline{\mathcal{C}}$ which satisfies (4), (5), and (6) of $\S 2$, with the minimum in (5) replaced by infimum.

THEOREM. Let the associated measures of the positive semi-group $\left(S_{t}\right)$ satisfy $p_{t}(\omega) \equiv 0$. Then Theorem 5.1 remains true with $S_{t}, \mathcal{C}, \mathcal{C}_{2}, G_{c}$ replaced by $\bar{S}_{t}$, $\overline{\mathcal{C}}, \overline{\mathcal{C}}_{2}, G$.

We shall prove only that the domain of the infinitesimal generator of $\left(\bar{S}_{t}\right)$ includes $\overline{\mathcal{C}}_{2}$. The rest of the proof follows easily from Theorem 5.1 and the estimates we produce.

First let us limit the behavior of $p_{t}$ near $\omega$. The $G$ occurring in Theorem 5.1 
now has the property that $G(\omega)=0$. Let $\delta$ be positive and $U$ a compact neighborhood of $\epsilon$ in $G$ such that $G(G-U)<\delta / 14$. There is a compact $V$ in $G$ whose interior includes $U$; let $\psi$ be a function in $\mathcal{C}_{2}$ which vanishes in $U$, takes on the constant value 1 in $G_{c}-V$, and elsewhere lies between 0 and 1 . Then

$$
\begin{aligned}
\delta / 14 & \geqq \int_{G^{-\{\epsilon\}}} \psi(\sigma) G(d \sigma) \\
& =\lim _{\lambda 0} \frac{1}{t} \int_{G^{-\{\epsilon\}}} \psi(\sigma) p_{t}(d \sigma) \\
& \geqq \limsup _{\lambda 0} \frac{1}{t} \int_{G^{-V}} p_{t}(d \sigma),
\end{aligned}
$$

so that $p_{t}(G-V)<t \delta / 7$ for small $t$.

Assume that for some $f$ in $\overline{\mathcal{C}}_{2}$ bounded by 1 the limit of $t^{-1}\left(\bar{S}_{t} f-f\right)$ does not exist in the metric of $\overline{\mathcal{C}}$. There are then a positive $\delta$ and sequences $\left(\tau_{n}\right)$, $\left(s_{n}\right),\left(t_{n}\right)$ such that $0<s_{n}, t_{n}<1 / n$ and

$$
\frac{1}{t_{n}}\left[\bar{S}_{t_{n}} f \cdot\left(\tau_{n}\right)-f\left(\tau_{n}\right)\right]-\frac{1}{s_{n}}\left[\bar{S}_{s_{n}} f \cdot\left(\tau_{n}\right)-f\left(\tau_{n}\right)\right]>\delta .
$$

We shall prove this leads to a contradiction.

The functions $f_{n} \equiv L_{\tau_{n}} f, X_{i} f_{n}, X_{i} X_{j} f_{n}$ have a common modulus of uniform continuity independent of $n$. We may therefore assume, passing to a subsequence if necessary, that as $n$ increases these functions converge uniformly on a compact neighborhood $V$ of $\epsilon$ (we suppose $V$ chosen as above) to a function $g$ and its derivatives $X_{i} g, X_{i} X_{j} g$. We alter $g$ on $G_{c}-V$ so that it becomes a function in $\mathcal{C}_{2}$ bounded by 1 . Write

$$
f_{n}=g+\sum c_{i}^{n} x_{i}+\sum c_{i j}^{n} x_{i} x_{j}+h_{n}
$$

where the $c_{i}^{n}, c_{i j}^{n}$ are chosen to make $h_{n}$ vanish at $\epsilon$ through terms of the second order. (The $x_{i}$, and later $\phi$, are those at the beginning of $\S 5$.) The $c_{i}^{n}$ and $c_{i j}^{n}$ clearly tend to 0 . Also $\left|h_{n}(\tau)\right|<\delta_{n} \phi(\tau)$ for $\tau$ in $V$, with $\delta_{n}$ tending to 0 , and $h_{n}(\tau)$ is bounded by 3 for large $n$.

We abbreviate $t^{-1}\left[\bar{S}_{t} u \cdot(\boldsymbol{\epsilon})-u(\boldsymbol{\epsilon})\right]$ to $[u, t]$ and $|[u, s]-[u, t]|$ to $[u, s, t]$. Then (1) and (2) imply, for large $n$, that

$$
\begin{aligned}
\delta< & {\left[f_{n}, s_{n}, t_{n}\right] } \\
\leqq & {\left[g, s_{n}, t_{n}\right]+\sum\left|c_{i}^{n}\right|\left[x_{i}, s_{n}, t_{n}\right]+\sum\left|c_{i j}^{n}\right|\left[x_{i} x_{j}, s_{n}, t_{n}\right] } \\
& +\delta_{n}\left|\left[\phi, s_{n}\right]\right|+\delta_{n}\left|\left[\phi, t_{n}\right]\right|+6 \delta / 7,
\end{aligned}
$$

where we have used the limitation on $p_{t}$ in estimating $\left[h_{n}, s_{n}, t_{n}\right]$ by the last 
line. Since $x_{i}, x_{i} x_{j}, \phi$ all belong to $\mathcal{C}_{2}$, every term in the last member except $6 \delta / 7$ tends to 0 . We have arrived at a contradiction; therefore $t^{-1}\left[\bar{S}_{t} f-f\right]$ converges uniformly on $G$ and the domain of the infinitesimal generator of $\left(\bar{S}_{t}\right)$ includes $\overline{\mathcal{C}}_{2}$.

If we had used the right uniform structure instead of the left the above proof would fail at the point of asserting that $L_{\tau_{n}} f, \cdots$ have a modulus of continuity independent of $n$. Theorem 5.1 can be modified to deal with the space of bounded functions uniformly continuous in the right uniform structure, but it is apparently difficult to pick out a sufficiently large recognizable class of functions included in the domain of the infinitesimal generator.

6.3. The Lévy formula now follows easily. Let $G$ be the reals and write $a, b, \sigma /\left(1+\sigma^{2}\right)$ for $a_{1}, a_{11}, x_{1}$ in (1). Denote by $e_{\xi}$ the character $e^{i \xi \sigma} ;$ it belongs to $\overline{\mathcal{C}}_{2}$ and

$$
A e_{\xi}=M e_{\xi} \cdot(0)=a i \xi-b \xi^{2}+\int_{\mathcal{G}^{-\{0\}}}\left(e^{i \xi \sigma}-1-\frac{i \xi \sigma}{1+\sigma^{2}}\right) G(d \sigma) .
$$

Moreover $\bar{S}_{t} e_{\xi} \cdot(\sigma)=e_{\xi}(\sigma) \bar{S}_{t} e_{\xi} \cdot(0)$, because $e_{\xi}$ is a character. Therefore

$$
\frac{d}{d t} \bar{S}_{t} e_{\xi} \cdot(0)=M \bar{S}_{t} e_{\xi} \cdot(0)=\left(A e_{\xi}\right) \bar{S}_{t} e_{\xi} \cdot(0) .
$$

Consequently $\int e^{i \xi \sigma} p_{t}(d \sigma) \equiv S_{t} e_{\xi} \cdot(0)=\exp \left(t A e_{\xi}\right)$, the result of Lévy.

7. Normal and self-adjoint semi-groups. When the measures associated with a positive semi-group $\left(S_{t}\right)$ are symmetric in the sense that $p_{t}=p_{t} \circ J$ -here $J$ is the involution $\tau \rightarrow \tau^{-1}$ extended to $G_{c}$ by setting $J(\omega)=\omega-$ the linear functional $A f=M f \cdot(\epsilon)$ can be written in a particularly simple form From the symmetry of $p_{t}$ follows that of $G$, so that in (1) of $\$ 5$ the integral may be written for $\tau=\epsilon$

$$
\frac{1}{2} \int_{G_{c}^{-\{\epsilon\}}}\left\{f(\sigma)+f\left(\sigma^{-1}\right)-2 f(\epsilon)-\sum X_{i} f \cdot(\epsilon)\left[x_{i}(\sigma)+x_{i}\left(\sigma^{-1}\right)\right]\right\} G(d \sigma) .
$$

The integrals $\int\left[x_{i}(\sigma)+x_{i}\left(\sigma^{-1}\right)\right] G(d \sigma)$ exist in the ordinary sense because $x_{i}(\sigma)+x_{i}\left(\sigma^{-1}\right)$ is of second order at $\epsilon$. This being so, we adsorb the last terms under the integral into $\Delta_{1}$ and write

$$
\begin{aligned}
A f= & \Delta_{1} f \cdot(\epsilon)+\Delta_{2} f \cdot(\epsilon)+\frac{1}{2} \int_{G^{-\{\epsilon\}}}\left[f(\sigma)+f\left(\sigma^{-1}\right)-2 f(\epsilon)\right] G(d \sigma) \\
& +G(\omega)[f(\omega)-f(\epsilon)] .
\end{aligned}
$$

It is clear that $A$ must take on the same values for $f$ and $f^{\prime}=f \circ J$. In view of the facts that $\Delta_{1} f^{\prime} \cdot(\epsilon)=-\Delta_{1} f \cdot(\epsilon)$, that $\Delta_{2}$ is a second order operator, and that the last two terms of (1) remain unchanged when $f^{\prime}$ replaces $f$-we must have $\Delta_{1}=0$; then $\Delta_{2}$ must satisfy $\Delta_{2} f^{\prime} \cdot(\epsilon)=\Delta_{2} f \cdot(\epsilon)$. So we may say:

If $p_{t} \circ J=p_{t}$ then 


$$
\begin{aligned}
A f= & \Delta_{2} f \cdot(\epsilon)+\frac{1}{2} \int_{G-\{\epsilon\}}\left[f(\sigma)+f\left(\sigma^{-1}\right)-2 f(\epsilon)\right] G(d \sigma) \\
& +G(\omega)[f(\omega)-f(\epsilon)],
\end{aligned}
$$

where the functional $f \rightarrow \Delta_{2} f \cdot(\epsilon)$ and the measure $G$ are invariant under $J$. Moreover, the semi-group $\left(S_{t}\right)$ is determined by the restriction of $A$ to those functions in $\mathcal{C}_{2}$ satisfying $f=f \circ J$.

Symmetry implies many interesting properties, both probabilistic and analytical, of the semi-group $\left(S_{t}\right)$. In the simplest case, that of discrete $G$ and $p_{t}(\omega)=0$, it is equivalent to symmetry $q_{t}(\sigma, \tau)=p_{t}\left(\sigma^{-1} \tau\right)=q_{t}(\tau, \sigma)$ of the transition probabilities. In more general situations it expresses itself most significantly in terms of a Markoff process inverse to the one introduced in \$5.3. To carry out this interpretation would lead us too far astray; instead we develop one of the analytical consequences.

7.1. Let $\left(S_{t}\right)$ be a positive semi-group whose associated measures are confined to $G$ and let $L^{2}$ be the Hilbert space of functions on $G$ which are square integrable with respect to the right-invariant Haar measure $\rho$. The equation

$$
T_{t} f \cdot(\tau)=\int_{G} f(\tau \sigma) p_{t}(d \sigma), \quad f \in L^{2},
$$

where the integral is taken in the sense of Bochner, defines $T_{t}$ as a linear transformation, with bound 1 , of $L^{2}$ into itself. The $T_{t}$ clearly form a semigroup converging to the identity as $t$ decreases to 0 ; so the infinitesimal generator $N$ is defined on a dense subset of $L^{2}$. It is easy to verify (because a subspace of $\mathcal{C}$ is dense in $L^{2}$ ) that $N$ coincides with the infinitesimal generator $M$ of the semi-group $\left(S_{t}\right)$ on the set of those functions $f$ in $\mathcal{C}_{2}$ which together with $X_{i} f$ and $X_{i} X_{j} f$ belong also to $L^{2}$ (and hence vanish at infinity).

We shall find conditions for the $T_{t}$ to be normal or self-adjoint. Since

$$
\iint \overline{g(\tau)} f(\tau \sigma) p_{t}(d \sigma) \rho(d \tau)=\iint f(\tau) \overline{g\left(\tau \sigma^{-1}\right)} p_{t}(d \sigma) \rho(d \tau), .
$$

the adjoint of $T_{t}$ is

$$
T_{t}^{*} f \cdot(\tau)=\int_{G} f(\tau \sigma) p_{t}^{\prime}(d \sigma),
$$

where $p_{t}^{\prime}=p_{t} \circ J$, a notation we shall employ for other measures also. A simple calculation shows that $T_{t}$ is normal if and only if $p_{t} * p_{t}^{\prime}=p_{t}^{\prime} * p_{t}$ and that $T_{t}$ is self-adjoint if and only if $p_{t}^{\prime}=p_{t}$.

7.2. Let us assume that all $T_{t}$ are normal and draw some conclusions about $N$. Zero is never a characteristic value of $T_{t}$, because $T_{t} f \rightarrow f$ as $t \rightarrow 0$; consequently $T_{t}=\int e^{t_{z}} E(d z)$ and the spectral measure $E$ is confined to the half-plane $R z \leqq 0$, because each $T_{t}$ has bound 1 . It follows that the infinitesi- 
mal generator $N$ is the normal transformation $\int z E(d z)$, defined for every $f$ for which the integral $\int|z|^{2}(E(d z) f, f)$ is finite. One can verify this in the following way: If the integral is finite then

$$
\left\|\frac{1}{t}\left(T_{t} f-f\right)-\int z E(d z) f\right\|^{2}=\int \frac{1}{t^{2}}\left|e^{t z}-1-t z\right|^{2}(E(d z) f, f),
$$

and the last integral tends to 0 with $t$ because the integrand does so while remaining less than $|z|^{2}$ for $R z \leqq 0$. Conversely, if $t^{-1}(T, f-f)$ converges then for some constant $K$

$$
\begin{aligned}
K & \geqq \limsup _{t \rightarrow 0}\left\|\frac{1}{t}\left(T_{t} f-f\right)\right\|^{2} \\
& =\limsup _{t \supset 0} \int \frac{1}{t^{2}}\left|e^{t z}-1\right|^{2}(E(d z) f, f) \\
& \geqq \int \lim _{\lambda \rightarrow 0} \frac{1}{t^{2}}\left|e^{t z}-1\right|^{2}(E(d z) f, f) \\
& =\int|z|^{2}(E(d z) f, f) .
\end{aligned}
$$

Thus $f$ belongs to the domain of $\int z E(d z)$ and the first argument applies.

7.3. We translate the conditions on the $p_{t}$ into ones on $M$ or $N$ by means of two remarks, the proofs of which we leave to the reader.

First, consider two positive semi-groups $\left(S_{t}^{1}\right)$ and $\left(S_{t}^{2}\right)$ with generators $M^{1}$ and $M^{2}$. For $S_{s}^{1}$ and $S_{t}^{2}$ to commute for all $s$ and $t$ it is necessary and sufficient that $M^{1} M^{2} f=M^{2} M^{1} f$ for all $f$ in $\mathcal{C}_{4}$.

Second, let $S_{t}, p_{t}, M, A$ have their usual meaning. Then $A^{\prime} f \equiv A(f \circ J)$ is obviously a linear functional defining the generator $M^{\prime}$ of a positive semigroup $\left(S_{t}^{\prime}\right)$. The measures associated with $\left(S_{t}^{\prime}\right)$ are precisely the $p_{t}^{\prime}=p_{t} \circ J$.

From these remarks it follows that the statements $p_{t} * p_{t}^{\prime}=p_{t}^{\prime} * p_{t}$ and $p_{t}^{\prime}=p_{t}$ for all $t$ are equivalent respectively to $S_{t} S_{t}^{\prime}=S_{t}^{\prime} S_{t}$ and $S_{t}^{\prime}=S_{t}$ for all $t$, and hence also to $M M^{\prime}=M^{\prime} M$ and $M=M^{\prime}$.

Let us consider the special case in which $A f=\int[f(\sigma)-f(\epsilon)] G(d \sigma)$ with $G$ a bounded measure on $G-\{\epsilon\}$. The $T_{t}$ are normal if and only if $G * G^{\prime}=G^{\prime} * G$. The necessity follows from the fact that $G$ is the weak limit of the measures $t^{-1} p_{t}$; the sufficiency, by noting that the infinitesimal generator is bounded and then using the same reasoning in $\S 7.2$. The commutativity of $M$ and $M^{\prime}$ in the general case, written in terms of the three components of $M$, is too cumbersome to be of use.

The condition $M=M^{\prime}$ is equivalent, we have seen, to the fact that $A$ can be written in the special form (2), with $G(\omega)=0$ if we are dealing with semi-groups on $L^{2}$. 
We have proved incidentally that the $\Delta_{1}$ and $\Delta_{2}$ in the resolution of the generator $N$ are always normal, the first "imaginary" and the second selfadjoint.

\section{Homogeneous Spaces}

8. Preliminaries. Let $K$ be a compact subgroup of the Lie group $G$ and $\mathcal{F}$ the homogeneous space of left cosets $\sigma K$. The group $K$ is denoted by $o$ when considered as a point of $\mathcal{X}$. If $\mathcal{X}$ is not compact let $\mathcal{X}_{c}$ be its one point compactification; the point $o^{\prime}$ at infinity is to be kept fixed under the action of $G$. If $\mathcal{X}$ is compact take $\mathcal{X}_{c}$ to be $\mathcal{X}$ itself. $\mathcal{C}$ is the Banach space of continuous functions on $\mathcal{X}_{c}$. For $\sigma$ in $G$ and $f$ in $\mathcal{C}$ the function $L_{\sigma} f$ is defined by $L_{\sigma} f \cdot(p)=f\left(\sigma^{-1} p\right)$.

Most of $\S 1$ carries over to homogeneous space. There are, however, a few points, notably the definition of $\mathcal{C}_{2}$ and the approximation of differential operators, which require elaboration. We shall use the same symbols as before except when the danger of ambiguity is too great.

8.1. Let $\mu$ be a finite measure on $\mathcal{X}_{c}$ with the property $\mu(\eta E)=\mu(E)$ for every $\eta$ in $K$. For each point $p$ of $\mathcal{X}$ we define a measure $\mu_{p}$ on $\mathcal{X}_{c}$ by setting $\mu_{p}(E)=\mu\left(\tau^{-1} E\right)$, where $\tau$ is an element of $G$ carrying $o$ into $p$; the measure $\mu_{p}$ depends only on $\mu$ and $p$, for two allowable choices of $\tau$ are related by $\tau^{\prime}=\tau \eta$ with $\eta$ in $K$. We define $\mu_{o^{\prime}}$ to be the measure whose total mass $\mu\left(\mathcal{X}_{c}\right)$ is concentrated at $o^{\prime}$.

By means of the family $\left(\mu_{p}\right)$ we associate with $\mu$ the continuous linear transformation

$$
R_{\mu} f \cdot(p)=\int_{\mathcal{X}_{c}} f(q) \mu_{p}(d q)
$$

of $\mathcal{C}$ into itself. Clearly $L_{\sigma} S_{\mu}=S_{\mu} L_{\sigma}$ for all $\sigma$ in $G$. Conversely, every continuous linear transformation of $\mathcal{C}$ into itself which commutes with all $L_{\sigma}$ can be so obtained from some measure invariant under $K$.

We define $\mu * \nu$ by

$$
\begin{array}{ll}
\mu * \nu \cdot(E)=\int_{\mathcal{X}} \nu_{p}(E) \mu(d p), & E \subset \mathcal{X}, \\
\mu * \nu \cdot\left(o^{\prime}\right)=\mu\left(o^{\prime}\right) \nu\left(\mathcal{X}_{c}\right)+\mu(\mathcal{X}) \nu\left(o^{\prime}\right), &
\end{array}
$$

so that $R_{\mu * \nu}=R_{\mu} R_{\nu}$. The remarks of $\$ 1.2$ concerning topology and continuity still apply.

8.2. Select arbitrarily a Riemann metric on $\mathcal{X}$ which is invariant under $G$; one can do this because $K$ is compact, and the particular choice is irrelevant at this point. Let $\delta(p)$ be the distance from $o$ to $p$ in this metric and let $z_{1}, \cdots, z_{l}$ be normal coordinates at $o$, valid in the neighborhood $\delta(p)<3 \alpha$ say. Let $\psi(u)$ be a twice differentiable function of the real variable $u$ which 
is 1 for $u \leqq \alpha^{2}$ and 0 for $u \geqq 4 \alpha^{2}$; define $x_{i}$ to be $\psi\left(\sum z_{j}^{2}\right) z_{i}$, and extend $x_{i}$ to a function in $\mathcal{C}$ by setting $x_{i}\left(o^{\prime}\right)=0$. We shall say the $x_{i}$ are modified normal coordinates at $o$.

An element $\eta$ of $K$ induces an isometry $p \rightarrow \eta p$ of $\mathcal{X}$ which leaves $o$ fixed. Since the $x_{i}$ are normal coordinates near $o$, so also are the functions $L_{\eta} x_{i}$. A moment's reflection shows that $L_{\eta} x_{j}=\sum c_{i j}(\eta) x_{i}$ with $\left(c_{i j}\right)$ an orthogonal matrix. Consequently, for every sufficiently differentiable function $f$ the quantities

$$
\sum_{i}\left(\frac{\partial f}{\partial x_{i}}\right)_{o}^{2}, \quad \sum_{i, j}\left(\frac{\partial^{2} f}{\partial x_{i} \partial x_{j}}\right)_{o}^{2}, \cdots
$$

are unchanged when the $x_{i}$ are replaced by the $L_{\eta} x_{i}$. This is true also of the function $f(o \mid q)$ of $q$

$$
f(o \mid q)=f(o)+\sum \frac{\partial f}{\partial x_{i}}(o) x_{i}(q), \quad q \in \mathcal{X}_{c} .
$$

The coordinates $x_{i}$ can be transported to any point $p$ of $\mathcal{X}$ by forming $y_{i} \equiv L_{\tau} x_{i}$ with $\tau o=p$. The $y_{i}$ depend upon the choice of $\tau$, but (according to the same reasoning as above) the quantities

$$
N_{1}(p, f)=\left\{\sum_{i}\left(\frac{\partial f}{\partial y_{i}}\right)_{p}^{2}\right\}^{1 / 2}, \quad N_{2}\left(p, f^{\prime}\right)=\left\{\sum_{i, j}\left(\frac{\partial^{2} f}{\partial y_{i} \partial y_{i}}\right)_{p}^{2}\right\}^{1 / 2}, \cdots
$$

and the function $f(p \mid q)=f(p)+\sum\left(\partial f / \partial y_{i}\right)_{p} y_{i}(q)$ of $q$ are the same for all allowable choices of $\tau$. We shall say that $f(p \mid q)$ is the first order part of $f$ at $q$; it depends not only on $f, p, q$ but also on the Riemannian metric and the function $\psi$.

$\mathcal{C}_{k}$ is the space comprising those functions $f$ in $\mathcal{C}$ with continuous derivatives on $\mathscr{X}$ for which the norm

$$
\|f\|_{k}=\max _{p \in \mathcal{X} f_{c}}|f(p)|+\sum_{1 \leqq r \leqq k} \sup _{p \in \mathcal{X} C} N_{r}(f, p)
$$

is finite and for which $N_{1}(p, f), \cdots, N_{k}(p, f)$ all tend to 0 as $p$ approaches $o^{\prime}$. Clearly $\mathcal{C}_{k}$ is a Banach space; it is also a dense subset of $\mathcal{C}$.

8.3. We shall say that an operator $T$ on $\mathcal{C}$ is invariant if its domain $E$ is invariant under the $L_{\sigma}$ and if $L_{\sigma} T f=T L_{\sigma} f$ for $\sigma$ in $G$ and $f$ in $E$. Such an operator is determined by the functional $B f=T f \cdot(o)$ on $E$,

$$
\begin{aligned}
& T f \cdot(p)=B\left(L_{\sigma} f\right), \\
& p \in \mathcal{X}, \sigma o=p, \\
& T f \cdot\left(o^{\prime}\right)=\lim _{p \rightarrow o} T f \cdot(p) .
\end{aligned}
$$

On the other hand, suppose that $E$ is a part of $\mathcal{C}$ invariant under all $L_{\sigma}$ and that $B$ is a functional on $E$. If $B$ is invariant in the sense that $B\left(L_{\eta} f\right)=B f$ for every $\eta$ in $K$ then the equations (1) define a function $T f$ on $\mathcal{X}_{c}$ provided the limit exists. In the examples we encounter it will be evident that the function 
lies in $\mathcal{C}$, so that (1) defines a transformation from $E$ to $\mathcal{C}$.

In particular take $E$ to be $C_{2}$ and

$$
B f=\sum a_{i} \frac{\partial f}{\partial x_{i}}(o)+\sum a_{i j} \frac{\partial^{2} f}{\partial x_{i} \partial x_{j}}(o), \quad a_{i j}=a_{j i},
$$

where the $x_{i}$ are the coordinates introduced in $\$ 8.2$. We have seen that

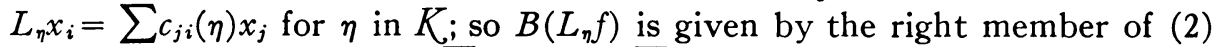
with $a_{i}$ and $a_{i j}$ replaced by $\sum a_{k} c_{k i}$ and $\sum a_{k l} c_{k i} c_{l j}$. For $B$ to lead to an invariant operator $\Delta$ it is necessary and sufficient that $a_{i}=\sum a_{k} c_{k i}(\eta)$ and $a_{i j}$ $=\sum a_{k l} c_{k i}(\eta) c_{l j}(\eta)$ for all $\eta$ in $K$. If this is so, $\Delta$ is a continuous linear transformation from the Banach space $\mathcal{C}_{2}$ to $\mathcal{C}$. Note that $\Delta f \cdot\left(o^{\prime}\right)=0$ according to the definition (1).

8.4. Consider a functional $B f=\sum a_{i j}\left(\partial^{2} f / \partial x_{i} \partial x_{j}\right)_{o}$ where the matrix $\left(a_{i j}\right)$ is positive semi-definite of rank $r$. After subjecting the $x_{i}$ to an orthogonal substitution we may write $B f=\sum_{1}^{r} \lambda_{i}\left(\partial^{2} f / \partial y_{1}^{2}\right)_{o}$. Let $p_{i}^{n}$ be the point $y_{j}\left(p_{l}^{n}\right)$ $=n^{-1} \delta_{i j}$ and $q_{i}^{n}$ the point $y_{j}\left(q_{i}^{n}\right)=-n^{-1} \delta_{i j}$. Observe that for a function of one real variable

$$
g^{\prime \prime}(0)-\frac{1}{v^{2}}[g(v)-2 g(0)+g(v)]=\frac{1}{v^{2}} \int_{-v}^{v}(v-|u|)\left[g^{\prime \prime}(0)-g^{\prime \prime}(u)\right] d u
$$

and that the right member is majorized by the oscillation of $g^{\prime \prime}(u)$ on the interval $-v \leqq u \leqq v$. Consequently the difference

$$
B f-n^{2} \sum_{1}^{r} \lambda_{i}\left[f\left(p_{i}^{n}\right)-2 f(o)+f\left(q_{i}^{n}\right)\right]
$$

is majorized by $\lambda \sum$ osc $\left(\partial^{2} f / \partial y_{i}^{2}\right)$, where $\lambda$ is the greatest of the $\lambda_{i}$ and the oscillation is taken over the neighborhood $\delta(p) \leqq l^{1 / 2} / n$ of $o$.

Let us assume further that $B L_{\eta} f=B f$ for every $\eta$ in $K$, so that $B$ defines an invariant operator $\Delta$. Denote by $\mu^{n}$ the measure which for $i=1, \cdots, r$ assigns to $p_{i}^{n}$ and $q_{i}^{n}$ each the measure $\lambda_{i} n^{2}$. Then the difference above may be written $B f-\int f(p) \mu^{n}(d p)$, if we assume, as we shall until equation (5), that $f$ vanishes at $o$. Observe now that

$$
B f-\int f(p) \mu^{n}(\eta d p)=B L_{\eta} f-\int L_{\eta} f \cdot(p) \mu^{n}(d p)
$$

and that there is a constant $\alpha$ such that $\lambda \sum$ osc $\left(\partial^{2} L_{\eta} f / \partial y_{i}^{2}\right)$ is majorized by $\alpha \sum$ osc $\left(\partial^{2} f / \partial x_{i} \partial x_{j}\right)$ provided all oscillations are over the same small neighborhood of $o$. Consequently

$$
\left|B f-\int f(p) \mu^{n}(\eta d p)\right| \leqq \alpha \sum_{\delta(p) \leqq l^{1 / 2} / n} \operatorname{osc}\left(\frac{\partial^{2} f}{\partial x_{i} \partial x_{j}}\right)
$$

for every $\eta$ in $K$. 
Take finally $\bar{\mu}^{n}$ to be the measure $\bar{\mu}^{n}(E)=\int \mu^{n}(\eta E) d \eta$, where $d \eta$ is the normalized Haar measure on $K$. Each $\bar{\mu}^{n}$ is invariant under $K$ and the difference $B f-\int f(p) \bar{\mu}^{n}(d p)$ is majorized by the right member of (4). Transporting to an arbitrary point $p$ of $\mathcal{X}$ and taking into account the definition of $\mathcal{C}_{2}$ we obtain

$$
\lim _{n \rightarrow \infty} \int[f(q)-f(p)] \bar{\mu}_{p}^{n}(d q)=\Delta f \cdot(p), \quad f \in \mathcal{C}_{2},
$$

the limit existing uniformly on $\mathcal{X}$. Since both members reduce to 0 for $p=o^{\prime}$, the convergence is actually in the metric of $\mathcal{C}$.

There is a similar approximation to $\Delta f$ for a $B$ of the general form (2) if the matrix $\left(a_{i j}\right)$ is positive semi-definite.

9. Positive semi-groups on $\mathcal{X}$. A semi-group $\left(S_{t}\right)$ of bounded linear transformations of $\mathcal{C}$ is positive if

$$
\begin{aligned}
& \lim _{t>0} S_{t} f=f, \\
& f \in \mathcal{C}, \\
& \min _{p \in \mathscr{X}_{c}} S_{t} f \cdot(p) \geqq \min _{p \in \mathscr{H}_{c}} f(p), \\
& t>0, f \in \mathcal{C}, \\
& L_{\sigma} S_{t}=S_{t} L_{\sigma}, \\
& t>0, \sigma \in G .
\end{aligned}
$$

It can be shown as in $\$ 2$ that a positive semi-group has the representation

$$
S_{t} f \cdot(p)=\int_{\mathcal{X}_{c}} f(q) Q_{t}(p, d q)
$$

where the Markoff transition probabilities $Q_{t}(p, E)$ are derived from a positive measure $P_{t}$ exactly as the $\mu_{p}$ were derived from $\mu$ in $\S 8.1$. The family $\left(P_{t}\right)_{0<t<\infty}$ has the following properties:

$$
\begin{aligned}
P_{t}\left(\mathscr{X}_{c}\right) & =1, & t>0, \\
\lim _{t \rightarrow 0} P_{t}(E) & =1, & \text { E a neighborhood of } o, \\
P_{t}(\eta E) & =P_{t}(E), & \eta \in K, \\
P_{s} * P_{t} & =P_{s+t}, & s, t>0 .
\end{aligned}
$$

In particular, $P_{t}$ is weakly continuous in $t$. Also, $P_{t}(\mathcal{X})$ and $P_{t}\left(o^{\prime}\right)$ vary continuously, for $P_{t}(\mathscr{X})$ is a bounded solution of the functional equation $u(s) u(t)=u(s+t)$.

Conversely a family $\left(P_{t}\right)$ of positive measures satisfying (5) through (8) leads to a positive semi-group when (4) is taken as a definition.

9.1. Let $x_{1}, \cdots, x_{l}$ be modified normal coordinates at $o$ and $\phi$ a function in $\mathcal{C}_{2}$ which is strictly positive on $\mathscr{X}_{c}-\{0\}$ and behaves like $\sum x_{\imath}^{2}$ near 0 .

THEOREM. The infinitesimal generator $M$ of a positive semi-group on $\mathcal{X}$ is 
an invariant operator which is defined at least on $\mathcal{C}_{2}$ and can be expressed at o as

(9) $M f \cdot(o)=\sum a_{i} \frac{\partial f}{\partial x_{i}}(o)+\sum a_{i j} \frac{\partial^{2} f}{\partial x_{i} \partial x_{j}}(o)+\int_{\mathcal{X}[c-\{o\}}[f(q)-f(o \mid q)] G(d q)$.

The matrix $\left(a_{i j}\right)$ is positive semi-definite; the linear functional

$$
B f \equiv \sum a_{i}\left(\partial f / \partial x_{i}\right)_{o}+\sum a_{i j}\left(\partial^{2} f / \partial x_{i} \partial x_{j}\right)_{o}
$$

satisfies $B L_{\eta} f=B f$ for all $\eta$ in $K$; and $G$ is a positive measure, $\int \phi G(d q)$ is finite, and $G(\eta E)=G(E)$ for $\eta$ in $K$.

Conversely, let $M$ be an invariant operator defined on $\mathcal{C}_{2}$ by (9) with $a_{i}, a_{i j}$, $G$ satisfying the conditions above. Then $M$ is the restriction to $\mathcal{C}_{2}$ of the infinitesimal generator of exactly one positive semi-group on $\mathcal{X}$.

The proof is so like the one of Theorem 5.1 it suffices to indicate the changes required beyond the replacement of $G$ by $\mathcal{X}, \epsilon$ by $o$ and so forth. $Y^{\prime} f, \mathcal{C}_{k}^{\prime},\|f\|^{\prime}$ being defined word for word as in $\$ 1.4$, but in terms of the new $\mathcal{C}$ and $L_{\sigma}$, the argument of $\$ 3.1$ shows that the restrictions of the $S_{t}$ to $\mathcal{C}_{2}^{\prime}$ form a semi-group to which $\$ 1.7$ applies. Let $z_{1}, \cdots, z_{l}$ be a fixed system of modified normal coordinates at $o$ and set $D_{i} f=\left(\partial f / \partial z_{i}\right)_{o}$ and

$$
D_{i j} f=\left(\partial^{2} f / \partial z_{i} \partial z_{j}\right)_{o}
$$

the lemma in $\$ 3.2$ remains valid - there is even the added simplicity that $D_{i j}=D_{j i}$. The function $\phi$ is chosen as before and $\mathcal{E}$ is the set of all functions in $\mathcal{C}$ which are twice continuously differentiable on $\mathcal{X}$. The argument now proceeds as in $\S 3$ through equation (4). At that point it is no longer necessary that the $x_{i}$ be in $\mathcal{D} \cap \mathcal{C}_{2}^{\prime}$; so we take them to be the coordinates $z_{i}$, which are in $\mathcal{C}_{2}$. Equation (4) of $\S 3$ becomes

$$
A f=\sum a_{i} D_{i} f+\sum a_{i j} D_{i j} f+\int_{\mathcal{S}(c-\{0\}}[f(q)-f(o \mid q)] G(d q) .
$$

Making use of the relation $A\left(L_{\eta} f\right)=A f$ for $\eta$ in $K$, one proves that $G$ is invariant under $K$ by considering those $f$ in $\mathcal{C}_{2}$ which vanish near $o$; at the same time one proves that $A$ determines $G$. It follows, on taking into account the properties of $f(o \mid q)$, that also $B f \equiv \sum a_{i} D_{i} f+\sum a_{i j} D_{i j} f$ satisfies the equation $B\left(L_{\eta} f\right)=B f$ for $\eta$ in $K$. The rest of $\S \S 3,4$ carries over to complete the proof.

It is easy to see that $M f \cdot\left(o^{\prime}\right)=0$.

9.2. The remarks of $\$ 5.2$ still apply. So does $\S 5.3$, with the exception that part of the probability interpretation is lost because there is no composition of random variables with values in $\mathcal{H}$. The elementary process corresponding to the Poisson may be described as follows: The process is specified by a set $F$ of the form $K p$ and a positive number $c$. Let $G$ be the measure on $F$ such 
that $G(F)=c$ and $G(\eta E)=G(E)$ for $\eta$ in $K$. The functional (9) for the process is $M f \cdot(o)=\int_{F}[f(q)-f(o)] G(d q)$.

9.3. As an example, take $\mathscr{Y C}$ to be a sphere, $G$ the group of rotations of $\mathcal{H}$, and $K$ the subgroup of $G$ leaving the point $o$ fixed. Now (9) simplifies a good deal. The group $K$ operates transitively on the unit tangent vectors to $\mathcal{X}$ issuing from $o$; so the term $\sum a_{i}\left(\partial f / \partial x_{i}\right)_{o}$ disappears (see $\left.\S 8.4\right)$. A similar argument shows that $\sum a_{i j}\left(\partial^{2} f / \partial x_{i} \partial x_{j}\right)_{o}$ reduces to a positive multiple of the ordinary Laplacian on $\mathcal{X}$. Also, $G$ must be a zonal distribution centered at $o$.

This example has been studied by Bochner [1] from the point of view of spectral analysis. Because of the zonal symmetry the manifold $\mathcal{X}$ may be replaced by the interval $[0,1]$; the convolution of invariant measures on $\mathcal{X}$ is commutative and when transported to $[0,1]$ makes the space of finite measures on $[0,1]$ a commutative ring.

The spectral theory often contains results that our method does not suggest. Here, for example, formulae are true which correspond formally to spheres of fractional dimension.

10. Translation to the group space. The preceding theorem enables us to characterize a weakly continuous semi-group $\left(p_{t}\right)$ of probability measures on $G_{c}$ which does not satisfy condition (2) of $\$ 2$.

10.1. There is certainly at least one probability measure $\mu$ and a sequence $s_{n}$ tending to 0 such that $p_{s_{n}}$ tends weakly to $\mu$. We also verify that $p_{s_{n}}(\omega)$ $\rightarrow \mu(\omega)$. Observe first that $p_{t}(G)$, being a bounded solution of the functional equation $u(s+t)=u(s) u(t)$, must either vanish identically or have the form $p_{t}(G)=e^{-c t}$. It is clear that $\mu(\omega)=p_{s_{n}}(\omega)=1$ under the first alternative, so that we need consider only the second. Let $A$ be an open set whose closure does not contain $\omega$ and $B$ a neighborhood of $\omega$ in $G_{c}$ such that $\sigma \tau \notin A$ for $\sigma$ in $A$ and $\tau$ in $B$. Then $p_{t+s}(A)=p_{t} * p_{s} \cdot(A) \leqq 1-p_{t}(A) p_{s}(B)$. Consequently

$$
\begin{aligned}
p_{t}(A) & =\liminf _{n \rightarrow \infty} p_{t+s_{n}}(A) \\
& \leqq 1-p_{t}(A) \underset{n \rightarrow \infty}{\limsup p_{s_{n}}(B)} \\
& \leqq 1-p_{t}(A) \mu(\omega) .
\end{aligned}
$$

By letting $A$ swell to $G$ and then letting $t$ decrease to 0 we obtain successively $p_{t}(G) \leqq 1-p_{t}(G) \mu(\omega)$ and $1 \leqq 1-\mu(\omega)$, from which we conclude that $\mu(\omega)=0$. So again $p_{s_{n}}(\omega)=1-e^{-c t} \rightarrow \mu(\omega)$.

It follows that

$$
p_{t} * \mu=\lim _{t \rightarrow \infty} p_{t} * p_{s_{n}}=\lim p_{t+s_{n}}=p_{t}, \quad t>0,
$$

by the remark at the end of $\S 1.1$; similarly $\mu * p_{t}=p_{t}$. If $\mu^{\prime}$ and $s_{n}^{\prime}$ are another such measure and sequence,

$$
\mu * \mu^{\prime}=\lim _{n \rightarrow \infty} p_{s_{n}} * \mu^{\prime}=\lim _{n \rightarrow \infty} p_{s_{n}}=\mu
$$


on the one hand, and $\mu * \mu^{\prime}=\mu^{\prime}$ on the other; hence $\mu=\mu^{\prime}$. A familiar argument now proves that $p_{t}$ tends to $\mu$ as $t$ decreases to 0 .

We have incidentally proved that $\mu * \mu=\mu$ and that $\mu(\omega)$ is either 0 or 1 .

10.2. We shall prove that if $\mu(\omega)=0$ then $\mu$ is the Haar measure of a compact subgroup of $G$. Let $K$ be the support of $\mu$ in $G$, that is to say, the support of $\mu$ in $G_{c}$ with $\omega$ deleted if necessary. Since $\mu$ is positive and finite the function $\mu(\sigma E)$, with $E$ a fixed compact set in $G$, is upper semi-continuous and approaches 0 as $\sigma$ approaches $\omega$. Consequently the supremum of $\mu(\sigma E)$ over the closed set $K$ is attained at some point $\tau$ of $K$. The facts that $\mu$ is positive and $\mu(K)$ is 1 and the equation

$$
\mu(\tau E)=\int_{K^{\mu\left(\sigma^{-1} \tau E\right) \mu(d \sigma)}}
$$

together imply that $\mu\left(\sigma^{-1} \tau E\right)=\mu(\tau E)$ almost everywhere on $K$ with respect to the measure $\mu$, therefore on a dense subset of $K$ (since $K$ is the support of $\mu)$. The upper semi-continuity of $\mu(\sigma E)$ now implies that the equality holds without exception on $K$.

It follows that $K$ is compact. For if not, we may take $E$ so that $\mu(E)$ is strictly positive and a sequence $\left(\sigma_{n}\right)$ in $K$ so that the sets $\sigma_{n}^{-1} \tau E$ are disjoint; but this leads to

$$
1=\mu(G) \geqq \sum_{n} \mu\left(\sigma_{n}^{-1} \tau E\right)=\sum_{n} \mu(\tau E)=\infty .
$$

It is easy now to prove that $K$ is a group. Note that $1=\mu(K) \geqq \mu\left(\sigma^{-1} K\right)$ for every $\sigma$ and that $\mu(K)=\int \mu\left(\sigma^{-1} K\right) \mu(d \sigma)$; the preceding argument shows that $\mu\left(\sigma^{-1} K\right)=\mu(K)$ for almost all $\sigma$ in $K$, hence without exception on $K$. This implies that $K$ is included in each $\sigma^{-1} K$, or that $K K$ is included in $K$. Consequently $K$, being compact, must be a group.

The equation $\mu\left(\sigma^{-1} \tau E\right)=\mu(\tau E)$, for a certain $\tau$ in $K$ and all $\sigma$ in $K$, shows that $\mu$ is invariant under $K$. So $\mu$ restricted to $K$ is the normalized Haar measure of $K$.

10.3. Let us consider $G$ as a fibre space over the homogeneous space $\mathscr{X}$ of cosets $\sigma K$. The natural projection $\pi$ extends to a continuous map of $G_{c}$ on $\mathcal{X}_{c}$ on setting $\pi(\omega)=o^{\prime}$. We identify the space of continuous functions on $\mathscr{X}_{c}$, denoted now by $\mathcal{C}\left(\mathcal{X}_{c}\right)$ to avoid ambiguity, with the subspace of $\mathcal{C}\left(G_{c}\right)$ comprising the functions satisfying $R_{\eta} f=f$ for every $\eta$ in $K$ by means of the isomorphism $g \leftrightarrow g \circ \pi$; under this identification $\mathcal{C}_{2}\left(\mathcal{X}_{c}\right)$ becomes $\mathcal{C}_{2}\left(G_{c}\right)$ $\cap \mathcal{C}\left(\mathscr{X}_{c}\right)$. There is a natural continuous projection $\Pi$,

$$
\Pi f \cdot(\tau)=\int_{K} f(\tau \eta) \mu(d \eta)
$$

of $\mathcal{C}\left(G_{c}\right)$ on $\mathcal{C}\left(\mathcal{X}_{c}\right)$ which maps $\mathcal{C}_{2}\left(G_{c}\right)$ on $\mathcal{C}_{2}\left(\mathscr{X}_{c}\right)$.

The transformations 


$$
\tilde{S}_{t} f \cdot(\tau)=\int_{G_{c}} f(\tau \sigma) p_{t}(d \sigma), \quad t>0, f \in \mathcal{C}\left(G_{c}\right),
$$

satisfy conditions (5) and (6) of $\$ 2$, but (4) only if $K$ reduces to $\{\epsilon\}$. However, $\Pi \widetilde{S}_{t}=\widetilde{S}_{t} \Pi=\widetilde{S}_{t}$ and $\tilde{S}_{t}$ converges strongly to $\Pi$ as $t$ decreases to 0 , so that the restrictions of the $\tilde{S}_{t}$ to $\mathcal{C}\left(\mathcal{X}_{c}\right)$ form a positive semi-group on $\mathcal{X}$.

Conversely, let $K$ be a compact subgroup of $G$ with Haar measure $\mu$ and let $\left(S_{t}\right)$ be a positive semi-group on the homogeneous space $\mathcal{H}$ of cosets $\sigma K$. If we define $\Pi$ as before and set $\widetilde{S}_{t}=S_{t} \Pi$, the $\widetilde{S}_{t}$ satisfy equations (5) and (6) of $\$ 2$ and $\widetilde{S}_{t}$ converges strongly to $\Pi$ as $t$ decreases to 0 . It is clear that the $\tilde{S}_{t}$ have the representation (1), that the $p_{t}$ in that representation form a weakly continuous semi-group of probability measures on $G_{c}$, that $p_{t}$ tends weakly to $\mu$ as $t$ decreases to 0 , and that $p_{t}(\omega)=\mu(\omega)=0$.

The two correspondences just described are inverses one to the other.

10.4. In the exceptional case of the limit measure $\mu$ concentrated at $\omega$ we make the following convention: The quotient space of $G_{c}$ modulo the equivalence relation $\sigma \omega=\tau \omega$ reduces to a single element $\bar{\omega}$. We consider $\{\bar{\omega}\}$ to be a homogeneous space on which $G$ acts trivially and $\{\omega\}$ to be a subgroup of $G$. The only positive semi-group on $\{\bar{\omega}\}$-that is, one satisfying (1) to (3) of $\$ 9$-is the one whose transformations are all the identity. With this convention the following statement is true without exception.

There is a natural one-to-one correspondence between the weakly continuous semi-groups of probability measures on $G_{c}$ and the pairs comprising a compact subgroup of $G_{c}$ and a positive semi-group on the corresponding homogeneous space. Thus the theorem in $\$ 9.1$ may be taken as a characterization of the weakly continuous semi-groups of probability measures on $G_{\text {c }}$.

\section{BIBLIOGRAPHY}

1. S. Bochner, Positive zonal functions on spheres, Proc. Nat. Acad. Sci. U.S.A. vol. 40 (1954) pp. 1141-1147.

2. K. Ito, Stochastic differential equations on a differentiable manifold, Nagoya Math. J. vol. 1 (1950) pp. 35-47.

3. P. Lévy, Sur les intégrales dont les éléments sont des variables aléatoires indépendantes, Annali R. Scuola Normale Superiore di Pisa series (2) vol. 3 (1934) pp. 337-366.

Cornell University,

ITHACA, N. Y. 